\title{
Poissonian products of random weights: Uniform convergence and related measures
}

Julien Barral

\begin{abstract}
The random multiplicative measures on $\mathbb{R}$ introduced in Mandelbrot $([22])$ are a fundamental particular case of a larger class we deal with in this paper. An element $\mu$ of this class is the vague limit of a continuous time measure-valued martingale $\mu_{t}$, generated by multiplying i.i.d. non-negative random weights, the $\left(W_{M}\right)_{M \in S}$, attached to the points $M$ of a Poisson point process $S$, in the strip $H=\left\{(x, y) \in \mathbb{R} \times \mathbb{R}_{+} ; 0<y \leq 1\right\}$ of the upper half-plane.

We are interested in giving estimates for the dimension of such a measure. Our results give these estimates almost surely for uncountable families $\left(\mu^{\lambda}\right)_{\lambda \in U}$ of such measures constructed simultaneously, when every measure $\mu^{\lambda}$ is obtained from a family of random weights $\left(W_{M}(\lambda)\right)_{M \in S}$, and $W_{M}(\lambda)$ depends smoothly upon the parameter $\lambda \in U \subset \mathbb{R}$.

This problem leads to study in several sense the convergence, for every $s \geq 0$, of the functions valued martingale $Z_{t}^{(s)}: \lambda \mapsto \mu_{t}^{\lambda}([0, s])$. The study includes the case of analytic versions of $Z_{t}^{(s)}(\lambda)$ where $\lambda \in \mathbb{C}^{n}$. The results make it possible to show in certain cases that the dimension of $\mu^{\lambda}$ depends smoothly upon the parameter.

When the Poisson point process is statistically invariant by horizontal translations, this construction provides the new non-decreasing multifractal processes with stationary increments $s \mapsto \mu([0, s])$ for which we derive limit theorems, with uniform versions when $\mu$ depends on $\lambda$.
\end{abstract}

2000 Mathematics Subject Classification: 28A33, 28A80, 60G18, 60G44, 60G55, 60G57. Keywords: Poisson point processes, Banach space valued martingales, random measures, Hausdorff dimension. 


\section{Introduction}

This paper follows a work on a new type of statistically self-similar multiplicative measures studied in Barral and Mandelbrot ([4]), and constructed as follows:

We are working in the upper half-plane $\left\{(x, y) \in \mathbb{R} \times \mathbb{R}_{+}^{*}\right\} ; x$ and $y$ are respectively denoted by the spatial and the scaling variable.

Let $\delta$ be a positive real number and let $\Lambda_{\delta}$ be the positive measure defined in the strip $H=\left\{(x, y) \in \mathbb{R} \times \mathbb{R}_{+} ; 0<y \leq 1\right\}$ by

$$
\Lambda_{\delta}(d x d y)=\frac{\delta}{2} \frac{d x d y}{y^{2}} \quad(\delta>0) .
$$

Then let $S$ be a Poisson point process with intensity $\Lambda_{\delta}$, and let $W$ be a positive integrable random variable. There is a natural way (see Section 2) to associate with each point $M \in S, W_{M}$, a copy of $W$, so that these copies are mutually independent and also independent of the random set $S$.

For $t \geq 1$ and $x \in \mathbb{R}$ define $C_{t}(x)=\left\{\left(x^{\prime}, y\right) \in H: 1 / t \leq y<1,\left|x^{\prime}-x\right| \leq y\right\}$ (see Figure 1).

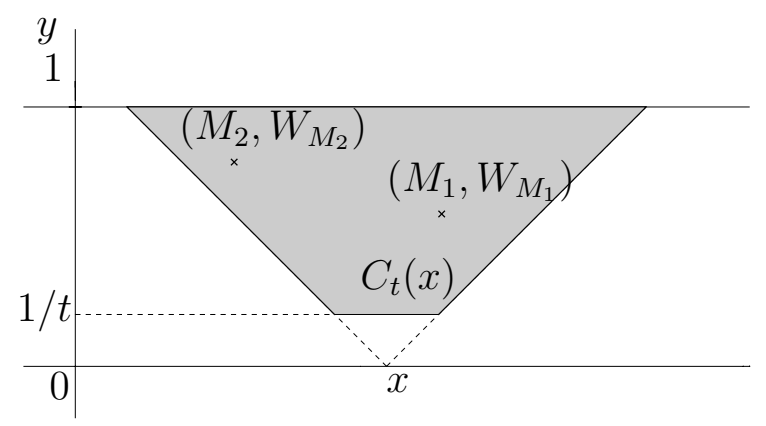

Figure 1: Truncated cone $C_{t}(x)$ containing two points $M_{1}$ and $M_{2}$ of the Poisson point process $S$.

Denote by $\ell$ the Lebesgue measure on $\mathbb{R}$.

With probability one, the family $\left(\mu_{t}\right)_{t \geq 1}$ of absolutely continuous positive measures whose densities are given by

$$
\frac{d \mu_{t}}{d \ell}(x)=t^{-\delta(\mathbb{E}(W)-1)} \prod_{M \in S \cap C_{t}(x)} W_{M}
$$

converges vaguely to a measure $\mu$ as $t \rightarrow \infty$. 
These measures were introduced in Mandelbrot ([22]) as an improvement of canonical cascade multifractals (CCM) (Mandelbrot ([21])) used to model intermittent phenomena, like turbulence, and recently used in mathematical finance (see Mandelbrot et al. ([23])).

On the one hand, they have important properties in common with CCM: because of the self similar properties of $\Lambda_{\delta}$ (see Section 3), the measure $\mu$ is statistically self similar; if $\mathbb{P}(W=1)<1$ and $\mu \neq 0, \mu$ is singular with respect to the Legesgue measure, $\mu$ is multifractal, and its multifractal function $\tau$ is determined explicitly in terms of the moments of $W$. Moreover, the number $-\tau^{\prime}(1)$ is $\mu$-almost everywhere the local dimension of $\mu$, so it is the smallest Hausdorff dimension of a Borel set carrying a piece of $\mu$, as well as the Hausdorff dimension of $\mu$. Thus $-\tau^{\prime}(1)$ gives a valuable information on the geometry of $\mu$.

On the other hand, while CCM possess an homogeneous tree structure, these measures do not involve a prescribed $b$-adic grid: they are statistically invariant under a continuous change of scale. For this reason, they bring in a great increase of realism.

Mandelbrot ([22]) calls these measures "multifractal products of cylindrical pulses". Indeed, the correct interpretation of the product of weights $\prod_{M \in S \cap C_{t}(x)} W_{M}$ is the following: for every $M \in S$, define (see Figure 2) the "cylindrical pulse" $P_{M}$ as the non negative function identically equal to $W_{M}$ on the interval $\left[x_{M}-y_{M}, x_{M}+y_{M}\right]$ and equal to 1 outside this interval. Then, for every $x \in \mathbb{R}$ and $t \geq 1$

$$
\prod_{M \in S \cap C_{t}(x)} W_{M}=\prod_{M \in S \cap\{1 / t \leq y\}} P_{M}(x) .
$$

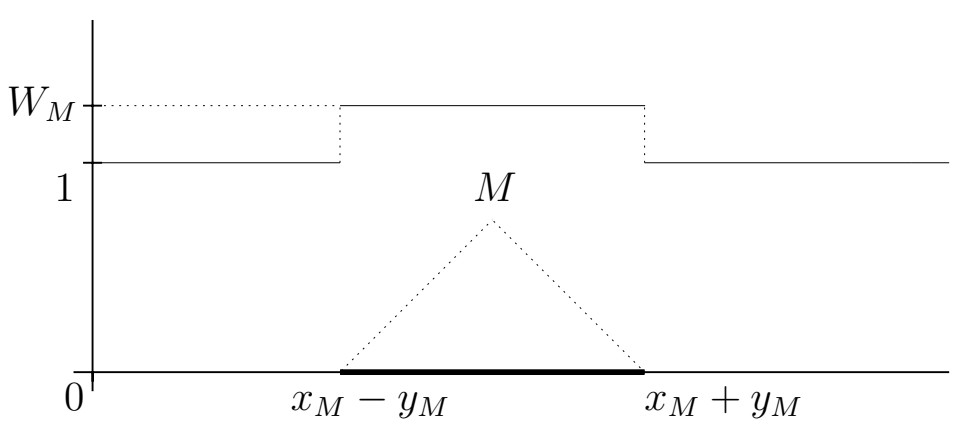

Figure 2: Cylindrical pulse associated with $\left(M, W_{M}\right)$.

Before continuing this introduction, let us indicate that the measures considered in this paper provide new examples of the general class of measures valued martingales defined in Kahane $([15])$; but the fine properties 
we are concerned with cannot be obtained from a simple application of the Kahane general results; as for other interesting examples of such measures like CCM (Kahane and Peyrière $([17])$ ) and the gaussian multiplicative chaos (Kahane ([14])) that inspired Kahane ([15]), they necessitate a specific study (see also examples given in Remark 1).

In this paper we are interested in what happens when the self-similarity properties of the intensity of the Poisson point process are relaxed. Indeed, the previous construction have a natural extension to the very large class of Poisson point processes in $H$ with Borel positive intensities; then it is natural to try to estimate the dimension of the non statistically self-similar associated limit measure, and to decide whether the limit measure is singular with respect to the Lebesgue measure when this dimension is equal to 1 .

This is done for measures associated with intensities invariant by translation in the spatial direction (i.e. the $x$-axis). Moreover we want to obtain these estimates almost surely simultaneously for uncountable families of such measures, by considering a fixed Poisson point process and random weights $W(\lambda)$ depending on a parameter $\lambda$ living in an uncountable set $U$.

The main reason to parametrize the problem is we have in mind to use these measures in some applications. It is fundamental to be able to make them dependent on a characteristic continuous parameter.

Without loss of generality, we study the restriction to $\mathbb{R}_{+}$of these measures.

The first difficulty is to define, with probability one, simultaneously such uncountable families $(\mu(\lambda))_{\lambda \in U}$. It turns out that it suffices to define simultaneously the total masses of the limit measures $\mu(\lambda)$ on any compact interval of the form $[0, s], s \geq 0$. This leads to study the convergence, as $t \rightarrow \infty$, of the functions valued martingales $\lambda \mapsto \mu_{t}(\lambda)([0, s])$, and to imbed them in some convenient spaces.

As the operations involved in the construction of the total masses do not require the weights to be positive (except, of course, when we consider families of measures) we shall deal with complex valued weights. Moreover, our general results of convergence do not involve any invariance property of the intensity $\Lambda$.

To be precise, consider $\Lambda$ a positive Borel measure on the strip $H$ (that is $\Lambda(K)<\infty$ for every compact subset $K$ of $H)$.

Let $S$ a be a Poisson point process with intensity $\Lambda$, and $W$ a complex integrable random variable. Then attach to each point $M \in S, W_{M}$, a copy of $W$, so that these copies are mutually independent and also independent of the random set $S$. 
For every $s \geq 0$, define the complex valued right continuous martingale $\left(Z_{t}^{(s)}\right)_{t \geq 1}$ with expectation $s$ by

$$
Z_{t}^{(s)}=\int_{0}^{s} e^{-\Lambda\left(C_{t}(x)\right)(\mathbb{E}(W)-1)} \prod_{M \in S \cap C_{t}(x)} W_{M} d x .
$$

The first main problem is to give conditions under which the martingale converges almost surely. We give sufficient conditions for the convergence in $L^{p}$ norm for $\left.\left.p \in\right] 1,2\right]$ (Theorems 1 and 6 ).

When $\Lambda$ is given by (1.1), the situation is the extension to $\mathbb{C}$ of the one described in the beginning of this section. After defining

$$
\tau_{W, \delta}(q)=1-q[\delta(\mathbb{E}(\Re(W))-1)+1]+\delta \mathbb{E}\left(|W|^{q}-1\right)
$$

for all $q \in \mathbb{R}$, the condition for the $L^{p}$ convergence is simply $\tau_{W, \delta}(p)<0$. Moreover, this condition is also necessary if $p=2$. This condition, although different, takes the same form as the one obtained in Biggins ([5], [6]) for the $L^{p}$ convergence of martingales in the branching random walk, i. e. extensions of complex CCM when the multiplicative cascade is made on a Galton-Watson tree.

The following three subsections detail the material of the paper. As a nice consequence of the construction, Section 1.3 provides new non-decreasing multifractal processes with stationary increments.

\subsection{Convergence of families of martingales}

Fix $s>0$ and denote $Z_{t}^{(s)}$ by $Z_{t}$ for all $t \geq 1$. Then consider $U$, an open subset of $\mathbb{R}$ (resp. $\mathbb{C}^{n}$ ), and a real (resp. complex) valued random function $\lambda \in U \mapsto W(\lambda)$ such that every $W(\lambda)$ is integrable. By taking mutually independent copies of $\lambda \mapsto W(\lambda)$, the $\lambda \mapsto W_{M}(\lambda)$ 's, associated to the $M$ 's in $S$ and chosen also to be independent of $S$, we obtain for each $\lambda \in U$ the martingale $\left(Z_{t}(\lambda)\right)_{t \geq 1}$ defined as $\left(Z_{t}\right)_{t \geq 1}$ but with the $W_{M}(\lambda)$ 's.

The questions we take an interest in are identical to those investigated in Joffe et al. ([13]), Biggins ([5], [6]) and Barral ([1], [3]) (see also references therein) for martingales in the branching random walk: we search to know whether the martingales $\left(Z_{t}(\lambda)\right)_{t \geq 1}$ converge almost surely simultaneously. If so, and if $\lambda \mapsto W(\lambda)$ has almost surely some regularity, we search to know whether $\lambda \mapsto Z(\lambda)=\lim _{t \rightarrow \infty} Z_{t}(\lambda)$ has almost surely some related regularity.

The Poisson point process structure involved in the construction is more complex than the one of the nodes in a Galton-Watson tree. This gives rise to serious mathematical complications in answering these questions. 
Our results are of the following kind:

a) when $U \subset \mathbb{C}^{n}$ and $\lambda \mapsto W(\lambda)$ is almost surely analytic, we show (Lemma 4 and Theorems 2 and 7) that the approach of Biggins ([6]) for martingales in the branching random walk can be adapted to the new family $Z_{t}(\lambda)$, and we obtain the almost sure uniform convergence of $\lambda \mapsto Z_{t}(\lambda)$ on the compact subsets of $U$. So the limit function $\lambda \mapsto Z(\lambda)$ is analytic.

b) When $U \subset \mathbb{R}$ and for every bounded open subinterval $I$ of $U$ the function $\lambda \mapsto W(\lambda)$ belongs to the Sobolev space $\mathcal{W}^{1, p_{I}}(I)$ (see Section 3 ) for some $\left.\left.p_{I} \in\right] 1,2\right]$, we give simple conditions under which the martingale $\lambda \mapsto Z_{t}(\lambda)$ converges almost surely in $\mathcal{W}^{1, p_{I}}(I)$ (Theorems 3.1) and 8.1)).

Then we refine the previous conditions by adding conditions on the second differences of $\lambda \mapsto W(\lambda)$ which make this process continuously differentiable, and as in Barral ([1]) for non-negative martingales in the branching random walk, we obtain the almost sure uniform convergence of $\lambda \mapsto Z_{t}(\lambda)$ on the compact subsets of $U$ to a continuously differentiable limit (Theorems $3.2)$ and 8.2)).

\subsection{Hölder exponent and dimension of the related measure}

When $W \geq 0$, for every $s \geq 0$, the martingale $\left(Z_{t}^{(s)}\right)_{t \geq 1}$ is non-negative and bounded in $L^{1}$. So the construction described in the beginning of the introduction can be extended to $\Lambda$. A non-negative measure $\mu$ on $\mathbb{R}_{+}$is obtained, and for every $s \geq 0$, almost surely $\mu([0, s])=Z^{(s)}=\lim _{t \rightarrow \infty} Z_{t}^{(s)}$.

Precisely, on $[0, s], \mu$ is the weak limit of the measures $\mu_{t}(t \geq 1)$ whose densities with respect to the Lebesgue measure are

$$
\frac{d}{d x} Z_{t}^{(x)}=e^{-\Lambda\left(C_{t}(x)\right)(\mathbb{E}(W)-1)} \prod_{M \in S \cap C_{t}(x)} W_{M}
$$

(see Section 2). We are interested in estimating the Hausdorff dimension of $\mu, \operatorname{dim} \mu$, i.e. the smallest Hausdorff dimension of a Borel set of full $\mu$-measure.

To do this, we assume that $\Lambda$ is invariant by translation in the spatial direction, and we denote by $\nu$ its projection on the scaling direction:

$$
\Lambda=\ell \otimes \nu
$$

It turns out that comparing $\Lambda\left(C_{s}(0)\right)$, the expected number of points in $S \cap C_{s}(0)$, with $\Lambda_{1}\left(C_{s}(0)\right)$, the expected number of points in $S \cap C_{s}(0)$ in the self-similar case when $\delta=1$, when $s \rightarrow \infty$, gives valuable information on the dimension of $\mu$. 
Define

$$
\begin{aligned}
& \delta_{1}=\lim _{t \rightarrow \infty} \inf _{s>t} \frac{\Lambda\left(C_{s}(0)\right)}{\Lambda_{1}\left(C_{s}(0)\right)}=\lim _{t \rightarrow \infty} \inf _{s>t} \frac{2 \int_{1 / s}^{1} y \nu(d y)}{\ln (s)} \\
& \delta_{2}=\lim _{t \rightarrow \infty} \sup _{s>t} \frac{\Lambda\left(C_{s}(0)\right)}{\Lambda_{1}\left(C_{s}(0)\right)}=\lim _{t \rightarrow \infty} \sup _{s>t} \frac{2 \int_{1 / s}^{1} y \nu(d y)}{\ln (s)} .
\end{aligned}
$$

For every integer $b \geq 2$ and every $t \in[1, \infty[$, define

$$
\delta_{2}(b, t)=\sup _{s \geq b t} \frac{\Lambda\left(C_{s}(0) \backslash C_{t}(0)\right)}{\Lambda_{1}\left(C_{s}(0) \backslash C_{t}(0)\right)}=\sup _{s \geq b t} \frac{2 \int_{1 / s}^{1 / t} y \nu(d y)}{\ln (s / t)} .
$$

Then define $\delta_{2}(b)=\lim \sup _{t \rightarrow \infty} \delta_{2}(b, t)$. The sequence $\left(\delta_{2}(b)\right)_{b \geq 2}$ is nonincreasing and we define

$$
\widehat{\delta}_{2}=\inf _{b \geq 2} \delta_{2}(b) .
$$

It is easily seen that $\delta_{1} \leq \delta_{2} \leq \widehat{\delta}_{2}$. We assume that $\sup _{0<\varepsilon \leq 1} \varepsilon \nu([\varepsilon, 1])<\infty$. Then $\widehat{\delta}_{2}<\infty$. Our results are the following:

a) (Theorem 9.i)) if $0<\delta_{1} \leq \delta_{2}, \mathbb{P}(W=1)<1$ and $\tau_{W, \widehat{\delta}_{2}}(p)<0$ for some $p \in] 1,2]$, then with probability one, conditionally on $\mu \neq 0$ : $0<-\tau_{W, \delta_{2}}^{\prime}(1) \leq$ $\operatorname{dim} \mu \leq-\tau_{W, \delta_{1}}^{\prime}(1)<1$ (in this paper, $\operatorname{dim} \mu$ denotes the smallest Hausdorff dimension of a Borel set of full $\mu$-measure).

The approach consists in estimating the limsup and the lim inf of the logarithmic density of $\mu, \mu$-almost everywhere.

In particular $\mu$ is singular with respect to the Lebesgue measure and if $\Lambda$ is asymptotically self-similar, i.e. if $\delta_{1}=\delta_{2}=\delta, \mu$ and the corresponding self-similar measure studied in Barral and Mandelbrot ([4]) borrow the same dimension. For example, this holds with $\widehat{\delta}_{2}=\delta_{2}=\alpha$ when $\nu=\sum_{n \geq \alpha} \delta_{\frac{\alpha}{n}}$ for some $\alpha>0$.

There is a progress with respect to the self-similar case because the proof uses properties weaker than the self-similarity of $\Lambda$.

b) (Theorem 9.ii)) if $\delta_{2}=0$ and $\tau_{W, \widehat{\delta}_{2}}(p)<0$ for some $\left.\left.p \in\right] 1,2\right]$, then with probability one, conditionally on $\mu \neq 0, \mu$ possesses a local Hölder exponent with respect to itself equal to 1 , and $\operatorname{dim} \mu=1$.

Then, our results on uniform convergence make it possible to give a continuous version of $a$ ) and $b$ ) when $\mu$ depends on a real parameter (Theorems 5 and 10).

c) We establish a necessary and sufficient condition for two such measures constructed simultaneously to be mutually singular. In particular we complete $b$ ) by a criterion to decide whether or not $\mu$ is absolutely continuous with respect to the Lebesgue measure when $W \neq 1$. (Theorem 11 and Corollary 2). 


\subsection{Limit theorems for the process with stationary increments $s \mapsto Z^{(s)}$ when $W \geq 0$}

When for every $s \geq 0, Z^{(s)}$ is almost surely the total mass of a piece of a measure like described in Subsection 1.2.a)b), we find that the $Z^{(s)}$ 's are in fact defined almost surely simultaneously. It is a consequence of the results on logarithmic densities described in Subsection 1.2.

Consequently, $\Lambda$ being invariant by translations in the spatial direction, we obtain the new type of non decreasing multifractal process $s \mapsto Z^{(s)}$, with stationary increments and with expectation $s \mapsto s$. (This multifractality is established in the self-similar case by the multifractal analysis of the measure $\mu$ performed in Barral and Mandelbrot ([4])).

We obtain for $\left(Z^{(s)}\right)_{s \geq 0}$ a result of the strong law of large numbers type: almost surely

$$
\lim _{s \rightarrow \infty} \frac{Z^{(s)}}{s}=1
$$

In the $L^{2}$ case, we also give a result of the law of the iterated logarithm type: almost surely

$$
\limsup _{s \rightarrow \infty} \frac{\left|Z^{(s)}-s\right|}{(2 s \log \log s)^{1 / 2}} \leq \sqrt{3}\left(\mathbb{E}\left[\left(Z_{1}-1\right)^{2}\right]\right)^{1 / 2} .
$$

These results (Theorem 12) have a uniform version (Theorem 13) when $Z^{(s)}$ depends on a real parameter $\lambda$.

Unfortunately, we are not able to prove that the $Z^{(s)}$ 's are defined almost surely simultaneously in the general case when $W \in \mathbb{C}$. But we conjecture that it is true. This result would provide a larger and very attractive class of multifractal processes with stationary increments.

The paper is organized as follows.

Section 2 describes definitions and notations used in the paper. Then, in order to simplify the reading of the paper, we develop the main ideas concerning the problem of convergence of martingales in Banach spaces in Section 3 devoted to the self-similar case. In Section 3 , when $\Lambda=\Lambda_{\delta}$, we study the $L^{p}$ convergence of the martingales $\left(Z_{t}^{(s)}\right)_{t \geq 1}$, their uniform convergence when they depend on a continuous parameter, and we give results on the local Hölder exponents and dimensions of the related statistically self-similar measures. In Section 4, we give extensions of results of Section 3 in the general case of non self-similar intensities. We also study the mutual singularity of the related measures. Section 5 is devoted to the behavior of $Z^{(s)}$ at $\infty$. 


\section{Definitions and notations}

\subsection{The martingales $\left(Z_{t}^{(s)}\right)_{t \geq 1}$}

Let $(\Omega, \mathcal{B}, \mathbb{P})$ be the probability space on which the random variables (r. v.) are defined in this paper.

Let $\Lambda$ be a positive Borel measure on the strip $H$ (see Section 1).

Let $W$ be a complex valued integrable random variable.

Let $\left\{E_{k}\right\}_{k \geq 1}$ be a partition of $H$ such that for all $k \geq 1,0<\Lambda\left(E_{k}\right)<\infty$. For every $k \geq 1$, denote by $\Lambda_{\mid E_{k}}$ the restriction of $\Lambda$ to $E_{k}$. Then choose $\left(M_{k, n}\right)_{n \geq 1}$ a sequence of $H$ valued r.v.'s with common law $\Lambda_{\mid E_{k}} / \Lambda\left(E_{k}\right)$, and $N_{k}$ a Poisson variable with parameter $\Lambda\left(E_{k}\right)$. Then choose $\left(W_{k, n}\right)_{k, n \geq 1}$ a sequence of copies of $W$.

Assume that the r.v.'s $M_{k, n}, W_{k, n}$ and $N_{k}, k, n \geq 1$, are independent of one another. Then define the Poisson point process with intensity $\Lambda$, $S=\left\{M_{k, n} ; 1 \leq k, 1 \leq n \leq N_{k}\right\}$ and for $M=M_{k, n} \in S$ define $W_{M}=W_{k, n}$.

For all $t \in \mathbb{R}_{+}$and $x \in \mathbb{R}_{+}$define the cone $C(x)=\left\{\left(x^{\prime}, y\right): 0 \leq y<1\right.$, $\left.\left|x^{\prime}-x\right| \leq y\right\}$ and the truncated cone at height $1 / t, C_{t}(x)=C(x) \cap\{1 / t \leq$ $y<1\}\left(C_{t}(x)=\emptyset\right.$ if $\left.t \leq 1\right)$ (see Figure 1 in Section 1 ). Then if $t \geq 1$ define

$$
Q_{t}(x)=Q_{W, t}(x)=e^{-\Lambda\left(C_{t}(x)\right)(\mathbb{E}(W)-1)} \prod_{M \in S \cap C_{t}(x)} W_{M} .
$$

Then for all $(s, t) \in \mathbb{R}_{+} \times[1, \infty[$ define

$$
Z_{t}^{(s)}=\int_{0}^{s} Q_{t}(x) d x
$$

and $F_{t}^{(s)}=\sigma\left(M, W_{M} ; M \in S \cap\left(\cup_{x \in[0, s]} C_{t}(x)\right)\right.$.

Proposition 1 For every $s \geq 0$, the stochastic process $\left(Z_{t}^{(s)}\right)_{t \geq 1}$ is a right continuous martingale with respect to the filtration $\left(F_{t}^{(s)}\right)_{t \geq 1}$, with expectation $s$.

Proof. Fix $s \geq 0$. By using the Fubini Theorem, it suffices to show that for every $x \in[0, s],\left(Q_{t}(x)\right)_{t \geq 1}$ is a right continuous martingale with respect to $\left(F_{t}^{(s)}\right)_{t \geq 1}$, with expectation 1 .

The right continuity and the measurability with respect to $F_{t}^{(s)}$ are consequences of the definition. The integrability of the martingale comes from a computation similar to the following. Fix $x \in[0, s]$ and $t \geq t^{\prime} \geq 1$. Then

$$
\mathbb{E}\left(Q_{t}(x) \mid F_{t^{\prime}}^{(s)}\right)=Q_{t^{\prime}}(x) e^{-\Lambda\left(C_{t}(x) \backslash C_{t^{\prime}}(x)\right)(\mathbb{E}(W)-1)} \mathbb{E}\left(\prod_{M \in S \cap C_{t}(x) \backslash C_{t^{\prime}}(x)} W_{M}\right) .
$$


Define $N=\# S \cap C_{t}(x) \backslash C_{t^{\prime}}(x)$ and $c=\Lambda\left(C_{t}(x) \backslash C_{t^{\prime}}(x)\right)$. By definition of a Poisson intensity measure and the independence of the $W_{M}$ 's, for all $k \geq 0$, $\mathbb{P}(N=k)=e^{-c} c^{k} / k !$ and

$$
\mathbb{E}\left(\prod_{M \in S \cap C_{t}(x) \backslash C_{t^{\prime}}(x)} W_{M} \mid N=k\right)=(\mathbb{E}(W))^{k} .
$$

So

$$
\mathbb{E}\left(\prod_{M \in S \cap C_{t}(x) \backslash C_{t^{\prime}}(x)} W_{M}\right)=\sum_{k=0}^{\infty}(\mathbb{E}(W))^{k} \mathbb{P}(N=k)=e^{\Lambda\left(C_{t}(x) \backslash C_{t^{\prime}}(x)\right)(\mathbb{E}(W)-1)} .
$$

\subsection{The related measure when $W \geq 0$.}

If $W \geq 0$, for all $t \in\left[1, \infty\right.$ [, let $\mu_{W, t}$ be the measure on $\mathbb{R}_{+}$whose density with respect to the Lebesgue measure $\ell$ is

$$
\frac{d \mu_{W, t}}{d \ell}(x)=e^{-\Lambda\left(C_{t}(x)\right)(\mathbb{E}(W)-1)} \prod_{M \in S \cap C_{t}(x)} W_{M} .
$$

Remark 1. When $W=0$ and $\Lambda$ is of the form $\ell \otimes \nu$, the measures $\mu_{0, t}$ are a particular case of those constructed in Kahane ([16]) for the study of the covering of the real line by the intervals $\left[x_{M}-y_{M}, x_{M}+y_{M}\right], M \in S$, when $\Lambda$ is invariant by translations in the spatial direction. Indeed Kahane ([16]) considers measures $\mu_{0, t}$ with $\mu_{0, t}(d x)=Q_{0, t}(x) \sigma(d x)$ where $\sigma$ is a positive measure on $\mathbb{R}$ or $\mathbb{R}_{+}$.

When $W$ is a positive constant and $\Lambda=\ell \otimes \sum_{n \geq 1} \delta_{\frac{\alpha}{n}}$ for some $\alpha>1$, these measures are considered in Fan ([12]) to study how many times a point is covered in Dvoretzky covering of the circle by random arcs when the sequence of length is $\alpha / n, n \geq \alpha$.

Both Kahane and Fan consider only an $L^{2}$ criterion to insure the limit measure to be non-degenerate.

Here weak convergence of measures on a compact subset $K$ of $\mathbb{R}_{+}$means weak*-convergence in the dual of $C(K)$, the space of real continuous functions on $K$. The general theory of Kahane ([15]) for positive measures valued martingales with discrete time parameter easily extends to right-continuous martingales. Consequently, the following holds.

Proposition 2 (Construction of the related measure) For every $n \in$ $\mathbb{N}$, with probability one, the measures $\mu_{W, t}^{(n)}, t \geq 1$, obtained by restriction to $[0, n]$ of the measures $\mu_{W, t}$, converge weakly to a measure $\mu_{W}^{(n)}$ as $t \rightarrow \infty$; moreover, $\mu_{W}^{(n)}(\{n\})=0$. Consequently, with probability one, there is an unique measure $\mu_{W}$ on $\mathbb{R}_{+}$such that the restriction of $\mu_{W}$ to $[0, n]$ is $\mu_{W}^{(n)}$ for all $n \in \mathbb{N}$. 
If $I$ is a non trivial compact subinterval of $\mathbb{R}_{+}$of length $|I| \leq 1$, define (see Figure 3)

$$
\begin{aligned}
T_{I} & =\{(x, y) \in H ; 0<y<|I|, \inf (I)-y \leq x \leq \sup (I)+y\} \\
B^{I} & =\{(x, y) \in H ;|I| \leq y<1, x \in(I-y) \cup(I+y)\} \\
T^{I} & =\{(x, y) \in H ;|I| \leq y<1, x \in[\sup (I)-y, \inf (I)+y]\}
\end{aligned}
$$

and $f_{I}$ the affine transformation on $\mathbb{R}$ which maps $\inf (I)$ onto 0 and $\sup (I)$ onto 1 .

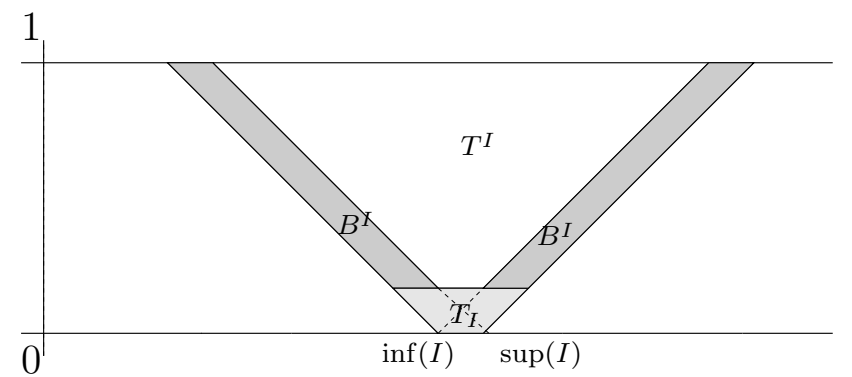

Figure 3:

For $t \geq 1$ and $x \in[0,1]$ define

$$
Q_{t, I}(x)=e^{-\Lambda\left(C_{\frac{t}{|I|}}(x) \backslash C_{\frac{1}{|I|}}(x)\right)(\mathbb{E}(W)-1)} \prod_{M \in S \cap C(x) \cap\left\{\frac{|I|}{t} \leq y<|I|\right\}} W_{M} .
$$

Then define on $I$ the following absolutely continuous measure with respect to the Lebesgue measure, $\mu_{W, t}^{I}$, whose density is

$$
\frac{d \mu_{W, t}^{I}}{d \ell}(x)=Q_{t, I}(x)
$$

If $G$ is a subset of $H$ with $\Lambda(G)<\infty$ define

$$
Q_{G}(x)=Q_{W, G}(x)=\prod_{M \in S \cap C(x) \cap G} W_{M}
$$

(this definition is also adopted in the case where $W \in \mathbb{C}$ ).

Proposition 3 With probability one, for every non trivial compact subinterval $I$ of $\mathbb{R}_{+}$of length $\leq 1$, the function $Q_{T^{I}}$ is a constant on $I$ and for all $t \geq 1 /|I|$

$$
\mu_{W, t}(I)=Q_{T^{I}} \int_{I} e^{-\Lambda\left(C_{\frac{1}{|I|}}(x)\right)(\mathbb{E}(W)-1)} Q_{B^{I}}(x) \mu_{W, t|I|}^{I}(d x)
$$


which can be written

$$
\mu_{W, t}(I)=e^{-\Lambda\left(C_{\frac{1}{|I|}}(0)\right)(\mathbb{E}(W)-1)} Q_{T^{I}} \int_{I} Q_{B^{I}}(x) \mu_{W, t|I|}^{I}(d x)
$$

if $\Lambda$ is invariant by translation in the spatial direction.

Moreover, with probability one, $\mu_{W, t}^{I}$ converges weakly to a measure $\mu_{W}^{I}$ as $t \rightarrow \infty$.

Notice that the choice of $\left(E_{k}, N_{k},\left(M_{k, n}\right)_{n \geq 1}\right)_{k \geq 1}$ and $\left(\left(W_{k, n}\right)_{n \geq 1}\right)_{k \geq 1}$ affects neither the probability distribution of the stochastic process

$$
\left(Q_{t}(x)\right)_{t \geq 1, x \in \mathbb{R}_{+}},
$$

nor those of the other random variables defined in this paper. Moreover, because of the properties of a Poisson point process, if $H_{1}, \ldots, H_{\ell}$ are mutually non overlapping subsets of $H$, the $\sigma$-algebras

$$
\sigma\left(M, W_{M} ; M \in S \cap H_{i}\right), \quad 1 \leq i \leq \ell,
$$

are mutually independent.

\section{The self-similar case}

In all the section

$$
\Lambda(d x d y)=\frac{\delta}{2} \frac{d x d y}{y^{2}} \quad(\delta>0)
$$

$\Lambda$ is the restriction to $H$ of the measure $\tilde{\Lambda}$ given by the same density on the whole upper half plane. $\tilde{\Lambda}$ is invariant by homothety with apex on the spatial variable axis and positive ratio, and it is also invariant by translation in the spatial direction. For this reason $\tilde{\Lambda}$ is called self-similar in Mandelbrot ([20]).

For $q \in \mathbb{R}$ define

$$
\tau_{W, \delta}(q)=1-q[\delta(\mathbb{E}(\Re(W))-1)+1]+\delta \mathbb{E}\left(|W|^{q}-1\right)
$$

and

$$
\theta_{W}(q)=\mathbb{E}\left(|W|^{q}-1\right)-q(\mathbb{E}(\Re(W))-1) .
$$

Note that $\tau_{W, \delta}(1) \geq 0$ and because of the convexity of $\theta_{W}, \theta_{W}(p) \geq 0$ for all $p \geq 1$. 
Theorem 1 ( $L^{p}$ convergence) $i$ ) If $\tau_{W, \delta}(p)<0$ for some $\left.\left.p \in\right] 1,2\right]$, then for every $s>0$, the martingale $\left(Z_{t}^{(s)}\right)_{t \geq 1}$ converges almost surely and in $L^{p}$ norm to a random variable $Z^{(s)}$ with mean $s$.

ii) If $p=2$, the previous condition is also necessary, and if it is fulfilled then for every $s \geq 0$

$$
\begin{array}{r}
\mathbb{E}\left(\left|Z^{(s)}\right|^{2}\right)=\int_{[0, s]^{2}}\left[\mathbf{1}_{\left\{\left|\frac{u-v}{2}\right|<1\right\}}(u, v) \mid\right. \\
\left.2 \frac{u-v}{2}\right|^{-\delta \theta_{W}(2)} e^{\delta\left(\left|\frac{u-v}{2}\right|-1\right)} \\
\left.+\mathbf{1}_{\left\{\left|\frac{u-v}{2}\right| \geq 1\right\}}(u, v)\right] d u d v .
\end{array}
$$

Remark 2. Because of the twice invariance of $\Lambda$, and also for the convenience of the reader, in all the section (except in the proof of Theorem 1.ii)) the proofs are given in the case $s=1$, i. e. for the martingale $Z_{t}:=Z_{t}^{(1)}$ with limit $Z=Z^{(1)}$.

Proof. $i$ ) Immediate consequence of Lemma 3 (see Section 3.4).

ii) It is Lemma 4 applied with $f=1$.

Now let $U$ be an open subset of $\mathbb{R}\left(\right.$ resp. $\mathbb{C}^{n}$ ) and $\lambda \mapsto W(\lambda)$ a random function from $U$ to $\mathbb{R}$ (resp. $\mathbb{C}$ ) such that for each $\lambda \in U$, the random variable $W(\lambda)$ is integrable and satisfies the hypothesis of Theorem 1.i).

Then choose, independently of the random set $S,\left(\lambda \mapsto W_{k, n}(\lambda)\right)_{k \geq 1, n \geq 1}$ a sequence of independent copies of $\lambda \mapsto W(\lambda)$. For every $s \geq 0$ and $\lambda \in U$, denote by $\left(Z_{t}^{(s)}(\lambda)\right)_{t \geq 1}$ the martingale defined as $\left(Z_{t}^{(s)}\right)_{t \geq 1}$ in Section 2 but with the $W_{k, n}(\lambda)$ 's instead of the $W_{k, n}$ 's. So for every $t \geq 1$ and $\lambda \in U$, $Z_{t}(\lambda)=Z_{t}^{(1)}(\lambda)$.

\subsection{Uniform convergence when $U \subset \mathbb{C}^{n}$ and $\lambda \mapsto W(\lambda)$ is analytic}

The following statement is of the same kind as the one of Biggins ([6]) about martingales in the branching random walk.

Theorem 2 Assume that $U \subset \mathbb{C}^{n}$ and fix $s>0$. Then assume that $\lambda \mapsto$ $W(\lambda)$ is almost surely analytic and that for every compact subset $K$ of $U$ there exists $p \in] 1,2]$ such that

$$
\sup _{\lambda \in K} \tau_{W(\lambda), \delta}(p)<0 .
$$

Then the family $\left(\lambda \mapsto Z_{t}^{(s)}(\lambda)\right)_{t \geq 1}$ converges uniformly on the compact subsets of $U$, almost surely and in mean, as $t \rightarrow \infty$, and its limit $\lambda \mapsto Z^{(s)}(\lambda)$ is almost surely analytic. 
Proof. Recall Remark 2. Lemma 3 makes it possible to use the approach of Biggins ([6]) for martingales in the branching random walk and, given an integer $b \geq 2$, with probability one, $\left(\lambda \mapsto Z_{b^{n}}(\lambda)\right)_{n \geq 1}$ converges uniformly on every compact subset $K$ of $U$ to an analytic function $\lambda \mapsto Z(\lambda)$.

Moreover, $\lambda \in K \mapsto Z(\lambda)$ is integrable in the separable Banach space $\left(C(K, \mathbb{C}),\|\cdot\|_{\infty}\right)$, that is $\mathbb{E}\left(\sup _{\lambda \in K}|Z(\lambda)|\right)<\infty$. To conclude we use the same approach as Joffe et al ([13]), except that we need a continuous time result for the convergence of martingales. For $t \geq 1$, let $\mathbb{F}_{t}$ denote the $\sigma$-algebra generated by the random continuous functions $\left(\lambda \in K \mapsto Z_{t^{\prime}}(\lambda)\right)$, $1 \leq t^{\prime} \leq t$. By the martingale property,

$$
\mathbb{E}\left(\lambda \in K \mapsto Z(\lambda) \mid \mathbb{F}_{t}\right)=\lambda \in K \mapsto Z_{t}(\lambda) .
$$

As the martingale $\left(\lambda \in K \mapsto Z_{t}(\lambda)\right)_{t \geq 1}$ is right continuous, the continuous time version of Proposition V-2-6 of Neveu ([24]) yields the uniform convergence, almost surely and in mean.

Remark 3. The uniform convergence of $\left(\lambda \mapsto Z_{b^{n}}^{(1)}(\lambda)\right)_{n \geq 1}$ was proved in Barral and Mandelbrot ([4]) in the particular case where $n=1$ and $W(\lambda)=W^{\lambda}$ for some positive integrable random variable $W$.

\subsection{Uniform convergence when $U \subset \mathbb{R}$ and $\lambda \mapsto W(\lambda)$ is differen- tiable}

If $I$ is an open subinterval of $\mathbb{R}$ and $p>1$ is a real number, $\mathcal{W}^{1, p}(I)$ denotes the Sobolev space of real functions

$$
\left\{u \in L^{p}(I) ; \exists g \in L^{p}(I) \text { such that } \int_{V} u \psi^{\prime}=-\int_{V} g \psi \quad \forall \psi \in C_{c}^{\infty}(I)\right\}
$$

equipped with the norm $\|u\|_{\mathcal{W}^{1, p}(I)}=\left\{\|u\|_{L^{p}(I)}^{p}+\|g\|_{L^{p}(I)}^{p}\right\}^{1 / p}$.

Recall that $\mathcal{W}^{1, p}(I)$ is a reflexive separable Banach space and that if $I$ is bounded then $u \in \mathcal{W}^{1, p}(I)$ if and only if $u \in L^{p}(I)$ and $u$ possesses an absolutely continuous version with almost everywhere a derivative $u^{\prime}$ such that $u^{\prime} \in L^{p}(I)$.

Define $\left(H_{1}\right)$ and $\left(H_{2}\right)$ the following sequences of assumptions.

$\left(\mathbf{H}_{\mathbf{1}}\right): U \subset \mathbb{R}, \mathbb{P}(W(\lambda) \neq 0)>0$ for every $\lambda \in U$, and $\lambda \mapsto W(\lambda)$ is almost surely absolutely continuous on the compact subintervals of $U$.

Moreover, for every bounded open subinterval $I$ of $U$ such that $\bar{I} \subset U$, there exists $\left.\left.p_{\bar{I}} \in\right] 1,2\right]$ and $C_{\bar{I}}>0$ such that 
i) the mapping $\lambda \mapsto \mathbb{E}\left(|W(\lambda)|^{p_{\bar{I}}}\right)$ is continuous on $\bar{I}$ and

$$
\sup _{\lambda \in \bar{I}} \tau_{W(\lambda), \delta}\left(p_{\bar{I}}\right)<0 ;
$$

ii)

$$
\forall\left(\lambda, \lambda^{\prime}\right) \in \bar{I}^{2}, \mathbb{E}\left(\left|W(\lambda)-W\left(\lambda^{\prime}\right)\right|^{p_{\bar{I}}}\right) \leq C_{\bar{I}}\left|\lambda-\lambda^{\prime}\right|^{p_{\bar{I}}} ;
$$

iii) there exists a deterministic Borel subset $D(I)$ of $I$ such that the Lebesgue measure of $I \backslash D(I)$ is null and almost surely $\lambda \in I \mapsto W(\lambda)$ is differentiable at each point of $D(I)$.

$\left(\mathbf{H}_{\mathbf{2}}\right)$ : The assumptions are those of $\left(H_{1}\right)$ except that $\left.i i i\right)$ is replaced by the following: there exist two positive functions $\psi$ and $\gamma$ on $\mathbb{R}_{+}^{*}$, both monotonically decreasing in a neighborhood of 0 , such that

$$
\frac{\psi(h)}{h} \text { and } \frac{\max \left(\gamma(h), h^{2 p_{\bar{I}}}\right)}{h^{p_{\bar{I}}+2} \psi^{p_{\bar{I}}}\left(\frac{h}{2}\right)}
$$

are integrable near 0 , and for all $(\lambda, h) \in \bar{I} \times \mathbb{R}_{+}$satisfying $\lambda+h \in \bar{I}$, and $\lambda-h \in \bar{I}$

$$
\mathbb{E}\left(|W(\lambda+h)+W(\lambda-h)-2 W(\lambda)|^{p_{\bar{I}}}\right) \leq C_{\bar{I}} \gamma(h)
$$

Theorem 3 Fix $s>0$.

1) Assume $\left(H_{1}\right)$. For every bounded open subinterval I of $U$ such that $\bar{I} \subset U$, the random functions $\lambda \in I \mapsto Z_{t}^{(s)}(\lambda), t \geq 1$, take values in the Sobolev space $\mathcal{W}^{1, p_{\bar{I}}}(I)$. Moreover, these functions converge almost surely, and in $L^{p_{\bar{I}}}$ norm in $\mathcal{W}^{1, p_{\bar{I}}}(I)$ as $t \rightarrow \infty$.

Consequently, the family $\left(\lambda \mapsto Z_{t}^{(s)}(\lambda)\right)_{t \geq 1}$ converges uniformly on the compact subintervals of $U$, almost surely and in mean as $t \rightarrow \infty$, and its limit $\lambda \mapsto Z^{(s)}(\lambda)$ is absolutely continuous on the compact subintervals of $U$.

2) Assume $\left(\mathrm{H}_{2}\right)$. With probability one, $\lambda \mapsto W(\lambda)$ and the functions $\lambda \mapsto$ $Z_{t}^{(s)}(\lambda), t \geq 1$, are continuously differentiable.

Moreover, the family $\left(\lambda \mapsto Z_{t}^{(s)}(\lambda)\right)_{t \geq 1}$ converges uniformly on the compact subintervals of $U$, almost surely and in mean as $t \rightarrow \infty$, and its limit $\lambda \mapsto Z^{(s)}(\lambda)$ is continuously differentiable.

Remark 4. 1) It is possible to give similar hypotheses under which for $n \geq 2$, the martingale converges in the Sobolev space $\mathcal{W}^{n, p_{\bar{I}}}(I)$ or in the space of $n$ times continuously differentiable functions, as it is done in this last space for martingales in the branching random walk in Biggins ([5]) and Barral ([1]). 
2) We cannot extend Theorem 3 to random functions of $n \geq 2$ variables because we need to control the supremum of such a function with the help of its gradient, and in general we need this gradient to be in $L^{p}(I)$ with $p>n$, which is incompatible with the estimates given in the fundamental Lemma 2 for $p \in] 1,2]$. Nevertheless, if the function $\lambda \mapsto W(\lambda)$ depends only on $\|\lambda\|$, the euclidean norm of $\lambda$, then the extension is immediate.

3) Conclusions of Theorem 3.1) still hold if $W \in \mathbb{C}$ and the elements of the Sobolev space take complex values. There is a difficulty to extend the conclusion of Theorem 3.2) to the complex case because of the second differences of the exponential function in the proof of Lemma 5 .

Proof of Theorem 3. Recall Remark 2. By assumptions $i$ ) and $i i)$ of $\left(H_{1}\right)$, we can write $U$ as a countable union of bounded open subintervals $I$ with $\bar{I} \subset U$, each $I$ being such that the assumptions of Corollary 1 (Section 3.4) are fulfilled for all $\left(W^{(1)}, W^{(2)}, W^{(3)}\right) \in\{W(\lambda) ; \lambda \in \bar{I}\}^{3}$, with $p=p_{\bar{I}}, \tau=$ $\sup _{\lambda \in \bar{I}}\left\{\tau_{W(\lambda), \delta}\left(p_{\bar{I}}\right)\right\}$, and $M^{\prime}$ uniformly bounded over $\left\{\left(W^{(1)}, W^{(2)}, W^{(3)}\right) \in\right.$ $\left.\{W(\lambda) ; \lambda \in \bar{I}\}^{3}\right\}$.

Proof of 1). Fix $I$ as described above. We have $\sup _{\lambda \in D(I)}\left\|\frac{d W}{d \lambda}(\lambda)\right\|_{p_{\bar{I}}}<\infty$ by assumptions $i$ i) and $i i i)$ of $\left(H_{1}\right)$ and the Fatou Lemma.

So $\mathbb{E}\left(\int_{\bar{I}}\left|\frac{d W}{d \lambda}(\lambda)\right|^{p_{\bar{I}}} d \lambda\right)<\infty$ by the Fubini Theorem and the boundedness of $I$. Moreover $\sup _{\lambda \in \bar{I}}\|W(\lambda)\|_{p_{\bar{I}}}<\infty$ by assumption. So a simple computation shows that $\lambda \in I \mapsto W(\lambda)$ is almost surely in $\mathcal{W}^{1, p_{\bar{I}}}(I)$.

Then, that $\lambda \in I \mapsto Z_{t}(\lambda)$ is in $\mathcal{W}^{1, p_{\bar{I}}}(I)$, absolutely continuous, and differentiable at every $\lambda \in D(I)$ follows from the assumption iii) of $\left(H_{1}\right)$, the construction of the functions $\lambda \mapsto Z_{t}(\lambda)$, and the fact that the product of two elements of $\mathcal{W}^{1, p_{\bar{I}}}(I)$ is also in $\mathcal{W}^{1, p_{\bar{I}}}(I)$ (see Brezis $([8])$ ).

Now, Lemma 3 and the martingale property yield $\sup _{\lambda \in \bar{I}, t>1}\left\|Z_{t}(\lambda)\right\|_{p_{\bar{I}}}<\infty$. Moreover, Corollary 1 (in Section 3.4) applied for every $\left(\lambda, \lambda^{\prime}\right) \in \bar{I}^{2}$ with $W^{(1)}=W^{(2)}=W(\lambda)$ and $W^{(3)}=W\left(\lambda^{\prime}\right)$ together with $\left.i i\right)$ in $\left(H_{1}\right)$ and the martingale property yield a constant $C^{\prime}>0$ such that

$$
\forall\left(\lambda, \lambda^{\prime}, t\right) \in \bar{I}^{2} \times\left[1, \infty\left[,\left\|Z_{t}(\lambda)-Z_{t}\left(\lambda^{\prime}\right)\right\|_{p_{\bar{I}}} \leq C^{\prime}\left|\lambda-\lambda^{\prime}\right| .\right.\right.
$$

It follows from (3.1) and the Fatou Lemma that

$$
\sup _{\lambda \in D(I), t \geq 1}\left\|\frac{d Z_{t}}{d \lambda}(\lambda)\right\|_{p_{\bar{I}}}<\infty .
$$

Then, it results from the previous remarks that $\left(\lambda \in I \mapsto Z_{t}(\lambda)\right)_{t>1}$ is a right continuous martingale in the separable reflexive Banach space $\mathcal{W}^{\overline{1}, p_{\bar{I}}}(I)$, and that it is bounded in $L^{p_{\bar{I}}}$ norm, in the sense that $\sup _{t \geq 1} \mathbb{E}(\| \lambda \in I \mapsto$ 
$\left.Z_{t}(\lambda) \|_{\mathcal{W}^{1, p} \bar{I}(I)}^{p_{\bar{I}}}\right)<\infty$; so it converges almost surely, and in $L^{p_{\bar{I}}}$ norm, since it is right continuous.

The reader is referred to Neveu ([24, Proposition V-2-8]), or Diestel and Uhl ([10, Corollary V.2.4 and Theorem V.2.8]), for results on the convergence of martingales with discrete time parameter taking values in Banach spaces. These results extend without difficulty to right continuous martingales taking values in a separable reflexive Banach space.

As $\lambda \mapsto W(\lambda)$ is chosen almost surely absolutely continuous, it is standard that the almost sure convergence of $\left(\lambda \in I \mapsto Z_{t}(\lambda)\right)_{t \geq 1}$ in $\mathcal{W}^{1, p_{\bar{I}}}(I)$ implies its almost sure uniform convergence on $\bar{I}$ to an absolutely continuous function $\lambda \in \bar{I} \mapsto Z(\lambda)$.

Moreover (3.2) and the fact that $\sup _{\lambda \in \bar{I}, t \geq 1}\left\|Z_{t}(\lambda)\right\|_{p_{\bar{I}}}<\infty$ give

$$
\mathbb{E}\left(\sup _{\lambda \in \bar{I}}|Z(\lambda)|\right)<\infty
$$

This yields the uniform convergence in mean by the same argument as in the proof of Theorem 2 .

As $U$ can be covered by a countable family of such intervals $I$, we have the global conclusion.

Proof of 2). Fix $I$ as above. If we show that $\lambda \in I \mapsto W(\lambda)$ is almost surely continuously differentiable, by construction it is also the case for all the functions $\lambda \in I \mapsto Z_{t}(\lambda)$, and as the assumption $\left.i i i\right)$ of $\left(H_{1}\right)$ is automatically fulfilled, we obtain the uniform convergence, almost surely and in mean, of $\lambda \in \bar{I} \mapsto Z_{t}(\lambda)$ to $\lambda \in \bar{I} \mapsto Z(\lambda)$.

By iii) of $\left(H_{2}\right)$, for all $(\lambda, h) \in \bar{I} \times \mathbb{R}_{+}$such that $\lambda+h$ and $\lambda-h$ are in $\bar{I}$, we have

$$
\mathbb{E}\left(|W(\lambda+h)+W(\lambda-h)-2 W(\lambda)|^{p_{\bar{I}}}\right) \leq C_{\bar{I}} \gamma(h) .
$$

By using the hypothesis on $\gamma$ and $\psi$, one shows easily (as in Barral ([1]) for another random function) that $\lambda \in \bar{I} \mapsto W(\lambda)$ satisfies almost surely the hypotheses of Lemma 7 (Section 3.4). Now it follows from Corollary 1 and assumption $\mathrm{ii}$ ) of $\left(\mathrm{H}_{2}\right)$ that there exists $C$ " $>0$ such that for all $(\lambda, h) \in$ $\bar{I} \times \mathbb{R}_{+}$such that $\lambda+h$ and $\lambda-h$ are in $\bar{I}$,

$$
\mathbb{E}\left(|Z(\lambda+h)+Z(\lambda-h)-2 Z(\lambda)|^{p_{\bar{I}}}\right) \leq C^{\prime \prime} \max \left(\gamma(h), h^{2 p_{\bar{I}}}\right) .
$$

We conclude that $\lambda \in \bar{I} \mapsto Z(\lambda)$ is almost surely continuously differentiable by using the hypothesis on $\gamma$ and $\psi$ and Lemma 7 again. 


\subsection{Dimensions of the related measures.}

Let $\mathcal{B}\left(\mathbb{R}_{+}\right)$denote the set of Borel subsets of $\mathbb{R}_{+}$, and for $B \in \mathcal{B}\left(\mathbb{R}_{+}\right)$, let $\operatorname{dim}(B)$ denote the Hausdorff dimension of $B$.

Assume that $\mathbb{P}(W>0)=1$. Among other results, the following is proved in Barral and Mandelbrot ([4]).

Theorem 4 If $\tau_{W, \delta}^{\prime}\left(1^{-}\right)<0$, then with probability one the measure $\mu_{W}$ has $\mathbb{R}_{+}$as support and for all $s \geq 0, \mathbb{E}\left(\mu_{W}([0, s])\right)=s$; if $\tau_{W, \delta}(p)<0$ for some $p>1$, then $\mu(B)>0 \Rightarrow \operatorname{dim}(B) \geq-\tau_{W, \delta}^{\prime}(1)$ for $B \in \mathcal{B}\left(\mathbb{R}_{+}\right)$. Moreover, $\operatorname{dim} \mu_{W}=-\tau_{W, \delta}^{\prime}(1)$.

Moreover, the measure $\mu_{W}$ is statistically self-similar in the following sense: for every non trivial compact subinterval $I$ of $\mathbb{R}_{+}$of length $\leq 1$ and $f \in$ $C(I)$, the r. v.'s $\int_{I} f(x) d \mu_{W}^{I}(x)$ and $|I| \int_{[0,1]} f \circ f_{I}^{-1}(x) d \mu_{W}(x)$ have the same distribution $\left(f_{I}^{-1}\right.$ and $\mu_{W}^{I}$ are defined in Section 2.2).

In particular $\left\|\mu_{W}^{I}\right\|$ has the same distribution as $|I|\left\|\mu_{W}([0,1])\right\|$.

Now consider the martingale studied in Theorem 3.2), but assume that with probability one $\lambda \mapsto W(\lambda)$ takes values in $] 0, \infty[$. Then, for every $\lambda \in U$ the hypothesis of Theorem 4 are fulfilled with $W(\lambda)$ and one obtains almost surely the associated measure $\mu_{W(\lambda)}$ and its dimension.

Due to the results obtained in Section 3.2, the measures $\mu_{W(\lambda)}$ are defined with probability one simultaneously for all $\lambda \in U$, and have all $\mathbb{R}_{+}$as support (the reader is referred to Section 4.2 for details). Then under additional conditions, the dimensions of these measures can be computed almost surely simultaneously:

Theorem 5 Assume that the assumptions of Theorem 3.2) are fulfilled and that for every compact subset $K$ of $U$, there exists $\eta>0$ such that

$$
\mathbb{E}\left(\left(\sup _{\lambda \in K} W(\lambda)\right)^{1+\eta}\right)+\sup _{\lambda \in K} \mathbb{E}\left(W^{-\eta}(\lambda)\right)<\infty .
$$

Then with probability one, for all $\lambda \in U$, for $\mu_{W(\lambda)}$-almost every $x \in \mathbb{R}_{+}$,

$$
\lim _{r \rightarrow 0^{+}} \frac{\log \mu_{W(\lambda)}([x-r, x+r])}{\log 2 r}=-\tau_{W(\lambda), \delta}^{\prime}(1) .
$$

Consequently, $\operatorname{dim} \mu_{W(\lambda)}=-\tau_{W(\lambda), \delta}^{\prime}(1)$. Moreover, $\mu_{W(\lambda)}(B)>0 \Rightarrow$ $\operatorname{dim}(B) \geq-\tau_{W(\lambda), \delta}^{\prime}(1)$ for $B \in \mathcal{B}\left(\mathbb{R}_{+}\right)$.

Remark 5. This statement generalizes an other one obtained for the family of Gibbs measures used in Barral and Mandelbrot ([4]) for the multifractal analysis of $\mu_{W}$. This family is associated with $\lambda \mapsto W^{\lambda}$, which is analytic. 
We leave the proof of Theorem 5 to the reader since it is a particular case of the one of Theorem 10 that we prefer to develop because it assumes properties weaker than the self-similarity (the reader will admit the following result obtained by mimicking the proofs of Theorem 5 in Barral ([2]) and Theorem 3 on moments of negative orders in Barral and Mandelbrot ([4]): $\left.\sup _{\lambda \in K} \mathbb{E}\left(Z_{1}^{-\eta / 2}(\lambda)\right)<\infty\right)$.

\subsection{Basic lemmas}

The assertions of the following lemma are established in the context where $W>0$ and $\Lambda=\Lambda_{\delta}$ in Barral and Mandelbrot ([4]), but they hold independently of the positivity of $W$ and the choice of $\Lambda$.

Lemma 1 Fix $G \subset H$ with $\Lambda(G)<\infty$.

i)

$$
\mathbb{E}\left(\prod_{M \in S \cap G} W_{M}\right)=e^{\Lambda(G)(\mathbb{E}(W)-1)} ;
$$

ii) For every compact subinterval I of $\mathbb{R}_{+}$of length $\leq 1$,

$$
\mathbb{E}\left(\sup _{x \in I}\left|Q_{W, B^{I}}(x)\right|\right) \leq e^{\Lambda\left(B^{I}\right)[\mathbb{E}(\max (1,|W|))-1]} .
$$

Lemma 2 (von Bahr and Esseen [26], Biggins [6]) Let $\left(U_{i}\right)_{i \geq 0}$ and $\left(V_{i}\right)_{i \geq 0}$ be two sequences of complex random variables such that $\sigma\left(U_{i} ; i \geq 0\right)$ and $\sigma\left(V_{i} ; i \geq 0\right)$ are independent and the $V_{i}$ 's are mutually independent. Assume that $\sum_{i>0} U_{i} V_{i}$ is almost surely defined and that for all $i \geq 0, V_{i}$ is integrable with mean 0 . Then for every $p \in] 1,2]$

$$
\mathbb{E}\left(\left|\sum_{i \geq 0} U_{i} V_{i}\right|^{p}\right) \leq 2^{p} \sum_{i \geq 0} \mathbb{E}\left(\left|U_{i}\right|^{p}\right) \mathbb{E}\left(\left|V_{i}\right|^{p}\right)
$$

Lemma 3 Fix $p \in] 1,2]$ and $b$ an integer $\geq 2$. Then

$$
\mathbb{E}\left(\left|Z_{b^{n+1}}-Z_{b^{n}}\right|^{p}\right) \leq \frac{12^{p}}{3} b^{\delta \theta_{W}(p)} b^{n \tau_{W, \delta}(p)} .
$$

Proof. For $n \geq 1$,

$$
\begin{aligned}
Z_{b^{n+1}}-Z_{b^{n}}= & \int_{0}^{1} e^{-\Lambda\left(C_{b^{n}}(x)\right)(\mathbb{E}(W)-1)} Q_{C_{b^{n}}(x)}(x) \times \\
& {\left[e^{-\Lambda\left(C_{b^{n+1}}(x) \backslash C_{b^{n}}(x)\right)(\mathbb{E}(W)-1)} Q_{C_{b^{n+1}}(x) \backslash C_{b^{n}}(x)}(x)-1\right] d x . }
\end{aligned}
$$


For $x \in[0,1]$, define

$$
\left\{\begin{array}{l}
u(x)=e^{-\Lambda\left(C_{b^{n}}(x)\right)(\mathbb{E}(W)-1)} Q_{C_{b^{n}}(x)}(x) \\
v(x)=e^{-\Lambda\left(C_{b^{n+1}}(x) \backslash C_{b^{n}}(x)\right)(\mathbb{E}(W)-1)} Q_{C_{b^{n+1}}(x) \backslash C_{b^{n}}(x)}(x)-1 .
\end{array}\right.
$$

Then, $Z_{b^{n+1}}-Z_{b^{n}}=\int_{\left[0, b^{-n}\right]}\left(\Gamma_{0}(x)+\Gamma_{1}(x)+\Gamma_{2}(x)\right) d x$, where for $i \in\{0,1,2\}$ and $x \in\left[0, b^{-n}\right]$,

$$
\Gamma_{i}(x)=\sum_{0 \leq 3 k+i<b^{n}} u\left(x+\frac{3 k+i}{b^{n}}\right) v\left(x+\frac{3 k+i}{b^{n}}\right) .
$$

Consequently

$\mathbb{E}\left(\left|Z_{b^{n+1}}-Z_{b^{n}}\right|^{p}\right) \leq 3^{p-1} b^{-n(p-1)} \int_{\left[0, b^{-n}\right]}\left[\mathbb{E}\left|\Gamma_{0}(x)\right|^{p}+\mathbb{E}\left|\Gamma_{1}(x)\right|^{p}+\mathbb{E}\left|\Gamma_{2}(x)\right|^{p}\right] d x$.

If $0 \leq k<b^{n}$ we denote $\left[\frac{k}{b^{n}}, \frac{k+1}{b^{n}}\right]$ by $J_{k}$. By construction, for $i \in\{0,1,2\}$ and $x \in\left[0, b^{-n}\right]$, in $\sum_{0 \leq 3 k+i<b^{n}} u\left(x+\frac{3 k+i}{b^{n}}\right) v\left(x+\frac{3 k+i}{b^{n}}\right)$, the $v\left(x+\frac{3 k+i}{b^{n}}\right)^{\prime} \mathrm{s}$ are of expectation 0 by Lemma 1.i), and these random variables are also independent because the $T_{J_{3 k+i}}$ 's (defined in Section 2) are mutually non overlapping, and they are independent of the $u\left(x+\frac{3 k+i}{b^{n}}\right)$ 's. So as $1<p \leq 2$, Lemma 2 yields

$$
\mathbb{E}\left(\left|\Gamma_{i}(x)\right|^{p}\right) \leq 2^{p} \sum_{0 \leq 3 k+i<b^{n}} \mathbb{E}\left(\left|u\left(x+\frac{3 k+i}{b^{n}}\right)\right|^{p}\right) \mathbb{E}\left(\left|v\left(x+\frac{3 k+i}{b^{n}}\right)\right|^{p}\right) .
$$

Lemma 1.i) applied with $|W|^{p}$ instead of $W$ and $G=C_{b^{n+1}}(x) \backslash C_{b^{n}}(x)$, together with a convexity inequality yield

$$
2^{p} \mathbb{E}\left(\left|v\left(x+\frac{3 k+i}{b^{n}}\right)\right|^{p}\right) \leq 2^{2 p} e^{\Lambda\left(C_{b^{n+1}}(x) \backslash C_{b^{n}}(x)\right) \theta_{W}(p)}=2^{2 p} b^{\delta \theta_{W}(p)} .
$$

Moreover, Lemma 1.i) applied with $|W|^{p}$ instead of $W$ and $G=C_{b^{n}}(x)$ yields

$$
\mathbb{E}\left(\left|u_{3 k+i}(x)\right|^{p}\right)=e^{\Lambda\left(C_{b^{n}}(x)\right) \theta_{W}(p)}=b^{n \delta \theta_{W}(p)} .
$$

So

$$
\mathbb{E}\left(\left|Z_{b^{n+1}}-Z_{b^{n}}\right|^{p}\right) \leq \frac{12^{p}}{3} b^{\delta \theta_{W}(p)} b^{n \tau_{W, \delta}(p)} .
$$

Lemma 4 Let $f$ be a bounded measurable function from $\mathbb{R}_{+}$to $\mathbb{C}$. For every $s \geq 0$ and $t \geq 1$ define $I_{t}^{(s)}=\int_{[0, s]} f(x) Q_{t}(x) d x$.

i)

$$
\mathbb{E}\left(\left|I_{t}^{(s)}\right|^{2}\right)=\int_{[0, s]^{2}} e^{\Lambda\left(C(u) \cap C(v) \cap\left\{y \geq \frac{1}{t}\right\}\right) \theta_{W}(2)} f(u) \bar{f}(v) d u d v .
$$


ii) For every $s \geq 0$

$$
\lim _{t \rightarrow \infty} \mathbb{E}\left(\left|I_{t}^{(s)}\right|^{2}\right)=\int_{[0, s]^{2}} e^{\Lambda(C(u) \cap C(v)) \theta_{W}(2)} f(u) \bar{f}(v) d u d v
$$

and $I_{t}^{(s)}$ converges almost surely and in $L^{2}$ norm if and only if the above integral is finite.

iii)

$$
\Lambda(C(u) \cap C(v))=-\delta \mathbf{1}_{\left\{\left|\frac{u-v}{2}\right|<1\right\}}(u, v)\left[\ln \left|\frac{u-v}{2}\right|-\left(\left|\frac{u-v}{2}\right|-1\right)\right],
$$

so if $\inf _{(u, v) \in[0, s]^{2}} \Re(f(u) \bar{f}(v))>0$ then the integral in ii) is finite if and only if $\delta \theta_{W}(2)<1$ i.e. $\tau_{W, \delta}(2)<0$.

Proof. $i$ ) It is a simple consequence of the Fubini Theorem, and Lemma 1.i) applied successively with $G=C(u) \cap C(v) \cap\left\{y \geq \frac{1}{t}\right\}=C_{t}(u) \cap C_{t}(v)$ and $W \bar{W}$ instead of $W$, with $G=C(u) \cap\left\{y \geq \frac{1}{t}\right\} \backslash C(v)$, and with $G=$ $C(v) \cap\left\{y \geq \frac{1}{t}\right\} \backslash C(u)$ and $\bar{W}$ instead of $W$.

ii) The existence and the value of the limit follow from an application of the monotone convergence Theorem. Then, an adaptation of the proof of Proposition 1 shows that $\left(I_{t}^{(s)}\right)_{t \geq 1}$ is a right continuous martingale bounded in $L^{2}$ if and only if the integral converges. So the result comes from the $L^{2}$-martingales convergence Theorem.

iii) It is a verification.

Now recall Remark 2 and let $\left(W^{(1)}, W^{(2)}, W^{(3)}\right)$ be a random integrable vector in $\mathbb{R}^{3}$. Independently of the random set $S$, choose $\left(W_{k, n}^{(1)}, W_{k, n}^{(2)}, W_{k, n}^{(3)}\right)_{k, n \geq 1}$ a sequence of independent copies of $\left(W^{(1)}, W^{(2)}, W^{(3)}\right)$. Then obtain by the initial construction three martingales $\left(Z_{t}^{(1)}\right)_{t \geq 1},\left(Z_{t}^{(2)}\right)_{t \geq 1}$ and $\left(Z_{t}^{(3)}\right)_{t \geq 1}$ respectively constructed with the $W_{k, n}^{(1)}$, the $W_{k, n}^{(2)}$ and the $W_{k, n}^{(3)}$. We denote by $Z^{(1)}, Z^{(2)}$ and $Z^{(3)}$ their respective limit when they exist.

Given an integer $b \geq 2$, define for every integer $k \geq 0, i \in\{1,2,3\}$ and $x \in \mathbb{R}_{+}$

$$
\begin{aligned}
a_{k}^{(i)}(x) & =e^{-\Lambda\left(C_{b^{k+1}}(x) \backslash C_{b^{k}}(x)\right)\left(\mathbb{E}\left(W^{(i)}\right)-1\right)} \prod_{M \in S \cap C_{b^{k+1}}(x) \backslash C_{b^{k}}(x)} W_{M}^{(i)} \\
& =b^{-\delta\left(\mathbb{E}\left(W^{(i)}\right)-1\right)} \prod_{M \in S \cap C_{b^{k+1}}(x) \backslash C_{b^{k}}(x)} W_{M}^{(i)} .
\end{aligned}
$$

For $p \geq 1$ define

$$
D_{p}\left(W^{(1)}, W^{(2)}, W^{(3)}\right)=\mathbb{E}\left(\left|W^{(1)}+W^{(2)}-2 W^{(3)}\right|^{p}\right)+\max _{1 \leq i, j \leq 3}\left[\mathbb{E}\left(\left|W^{(i)}-W^{(j)}\right|^{p}\right)\right]^{2} .
$$


Lemma 5 Assume that for some $p \geq 1,0<\mathbb{E}\left(\left|W^{(i)}\right|^{p}\right)<\infty, i \in\{1,2,3\}$. Fix $\rho>\max _{1 \leq i, j \leq 3} \frac{\mathbb{E}\left(\left|W^{(i)}\right|^{p}\right)}{\mathbb{E}\left(\left|W^{(j)}\right|^{p}\right)}$. Then define

$$
\left\{\begin{array}{l}
M=\left(1+\rho \max _{1 \leq i \leq 3} \mathbb{E}\left(\left|W^{(i)}\right|^{p}\right)\right)^{2} \\
\varepsilon=\max _{1 \leq i \leq 3} \delta \mathbb{E}\left(\left|W^{(i)}\right|^{p}\right)(\rho-1)+p \max _{1 \leq i, j \leq 3}\left[\delta \mathbb{E}\left(W^{(i)}-W^{(j)}\right)\right]
\end{array}\right.
$$

and

$$
\tau=\max _{1 \leq i \leq 3} \tau_{W^{(i)}, \delta}(p) .
$$

There exists a universal constant $C_{0}>0$ such that for all integers $b \geq 2$ and $k \geq 0$, and all $x \in[0,1]$

$$
\begin{array}{r}
\mathbb{E}\left(\left|a_{k}^{(1)}(x)+a_{k}^{(2)}(x)-2 a_{k}^{(3)}(x)\right|^{p}\right) \leq C_{0} \max \left(1, \delta^{4} \log ^{4}(b)\right) M b^{\tau+\varepsilon+p-1} \\
. D_{p}\left(W^{(1)}, W^{(2)}, W^{(3)}\right) .
\end{array}
$$

Proof. Fix $b \geq 2, k \geq 0$ and $x \in[0,1]$. If $n \geq 1$, conditionally on $\# S \cap$ $C_{b^{k+1}}(x) \backslash C_{b^{k}}(x)=n$, there exist $n$ independent copies of $\left(W^{(1)}, W^{(2)}, W^{(3)}\right)$, the $\left(W_{i}^{(1)}, W_{i}^{(2)}, W_{i}^{(3)}\right)^{\prime}$ 's, also independent of $S$, such that

$$
a_{k}^{(1)}(x)+a_{k}^{(2)}(x)-2 a_{k}^{(3)}(x)=\alpha_{1} \beta_{1}+\alpha_{2} \beta_{2}+\alpha_{3} \beta_{3}
$$

with

$$
\left\{\begin{array}{l}
\alpha_{1}=b^{-\delta\left(\mathbb{E}\left(W^{(1)}\right)-1\right)}+b^{-\delta\left(\mathbb{E}\left(W^{(2)}\right)-1\right)}-2 b^{-\delta\left(\mathbb{E}\left(W^{(3)}\right)-1\right)} \\
\alpha_{2}=b^{-\delta\left(\mathbb{E}\left(W^{(1)}\right)-1\right)}-b^{-\delta\left(\mathbb{E}\left(W^{(2)}\right)-1\right)} \\
\alpha_{3}=b^{-\delta\left(\mathbb{E}\left(W^{(1)}\right)-1\right)} \\
\beta_{1}=\prod_{i=1}^{n} W_{i}^{(3)}, \beta_{2}=\prod_{i=1}^{n} W_{i}^{(2)}-\prod_{i=1}^{n} W_{i}^{(3)} \\
\beta_{3}=\prod_{i=1}^{n} W_{i}^{(1)}+\prod_{i=1}^{n} W_{i}^{(2)}-2 \prod_{i=1}^{n} W_{i}^{(3)}
\end{array}\right.
$$

By using the differentiability of the exponential function one obtains

$$
\left\{\begin{array}{l}
\left|\alpha_{1}\right|^{p} \leq \delta^{2 p} \log ^{2 p}(b) b^{-p \delta \mathbb{E}\left(\left(W^{(3)}\right)-1\right)} b^{\varepsilon_{1}} D_{p}\left(W^{(1)}, W^{(2)}, W^{(3)}\right) \\
\left|\alpha_{2}\right|^{p} \leq\left.\delta^{p} \log ^{p}(b)\left|b^{-p \delta \mathbb{E}\left(\left(W^{(3)}\right)-1\right)} b^{\varepsilon_{1}}\right| \mathbb{E}\left(W^{(1)}-W^{(2)}\right)\right|^{p} \\
\alpha_{3}^{p} \leq b^{-p \delta \mathbb{E}\left(W^{(3)}-1\right)} b^{\varepsilon_{1}}
\end{array}\right.
$$

with $\varepsilon_{1}=p \max _{1 \leq i, j \leq 3}\left[\delta \mathbb{E}\left(W^{(i)}-W^{(j)}\right)\right]$ 
Moreover $\mathbb{E}\left(\left|\beta_{1}\right|^{p}\right)=\mathbb{E}\left(\left|W^{(3)}\right|^{p}\right)^{n}$, and by using the identity

$$
\begin{aligned}
\prod_{i=1}^{n} a_{i}+ & \prod_{i=1}^{n} b_{i}-2 \prod_{i=1}^{n} c_{i}=\sum_{i=1}^{n}\left(a_{i}+b_{i}-2 c_{i}\right) \prod_{j=1}^{i-1} c_{j} \prod_{j=i+1}^{n} a_{j} \\
& +\sum_{i=1}^{n} \prod_{j=1}^{i-1} c_{j}\left(b_{i}-c_{i}\right)\left[\sum_{j=i+1}^{n}\left(b_{j}-a_{j}\right) \prod_{j^{\prime}=i+1}^{j-1} a_{j^{\prime}} \prod_{j^{\prime}=j+1}^{n} b_{j^{\prime}}\right]
\end{aligned}
$$

together with the convexity of $x \mapsto x^{p}$ on $\mathbb{R}_{+}$one obtains

$$
\mathbb{E}\left(\left|\beta_{2}\right|^{p}\right) \leq n^{p}\left(\mathbb{E}\left(\left|W^{(3)}\right|^{p}\right)\right)^{n-1} \rho^{n-1} \mathbb{E}\left(\left|W^{(2)}-W^{(3)}\right|^{p}\right)
$$

and

$$
\begin{array}{r}
\mathbb{E}\left(\left|\beta_{3}\right|^{p}\right) \leq 2^{p-1} n^{p}\left(\mathbb{E}\left(\left|W^{(3)}\right|^{p}\right)\right)^{n-2} \rho^{n-2}\left[\mathbb{E}\left(\left|W^{(3)}\right|^{p}\right) \rho+(n-1)^{p}\right] \\
. D_{p}\left(W^{(1)}, W^{(2)}, W^{(3)}\right) .
\end{array}
$$

Define $\alpha=\mathbb{E}\left(\left|W^{(3)}\right|^{p}\right) \rho$ and $\alpha_{n}=\alpha^{n}+3 n^{2} \alpha^{n-1}+2 n^{2}(n-1) \alpha^{n-2}$ for $n \geq 1$. Since $p \leq 2$, it follows from the previous computations that

$$
\begin{aligned}
\mathbb{E}\left(\mid a_{k}^{(1)}(x)\right. & \left.+a_{k}^{(2)}(x)-\left.2 a_{k}^{(3)}(x)\right|^{p} \mid \# S \cap C_{b^{k+1}}(x) \backslash C_{b^{k}}(x)=n\right) \\
& \leq \max \left(1, \delta^{4} \log ^{4}(b)\right) b^{-p \delta \mathbb{E}\left(\left(W^{(3)}\right)-1\right)+\varepsilon_{1}} \alpha_{n} D_{p}\left(W^{(1)}, W^{(2)}, W^{(3)}\right) .
\end{aligned}
$$

Taking in account the fact that $\mathbb{P}\left(\# S \cap C_{b^{k+1}}(x) \backslash C_{b^{k}}(x)=n\right)=b^{-\delta \frac{(\delta \log (b))^{n}}{n !}}$ yields a universal constant $C_{0}>0$ such that

$$
\begin{aligned}
\mathbb{E}\left(\mid a_{k}^{(1)}(x)+a_{k}^{(2)}(x)-\right. & \left.2 a_{k}^{(3)}(x)\right|^{p} \mid \leq C_{0} \max \left(1, \delta^{4} \log ^{4}(b)\right)(1+\alpha)^{2} \times \\
& \times b^{-p \delta \mathbb{E}\left(\left(W^{(3)}\right)-1\right)+\varepsilon_{1}+\delta(\alpha-1)} D_{p}\left(W^{(1)}, W^{(2)}, W^{(3)}\right),
\end{aligned}
$$

and the conclusion follows from the definitions of $M, \varepsilon$ and $\tau$.

Lemma 6 Assume the hypotheses and notations of Lemma 5. Assume, moreover, that $p \in] 1,2]$ and $\max _{1 \leq i, j \leq 3} \mathbb{E}\left(\left|W^{(i)}-W^{(j)}\right|^{p}\right) \leq 1$.

Define $M^{\prime}=\left(\max \left(1, \delta^{4} \log ^{4}(b)\right) M b^{\varepsilon}\right)^{2 / p}$. There exists a universal constant $C_{0}^{\prime}$ such that for all integers $b \geq 2$ and $n \geq 0$,

$$
\left\|Z_{b^{n}}^{(1)}+Z_{b^{n}}^{(2)}-2 Z_{b^{n}}^{(3)}\right\|_{p} \leq C_{0}^{\prime} M^{\prime} b^{(p-1) / p} \sum_{m=0}^{n-1}(1+m)^{2} b^{\frac{m+1}{p} \tau} D_{p}\left(W^{(1)}, W^{(2)}, W^{(3)}\right)^{1 / p} .
$$

Corollary 1 Under the assumptions and notations of Lemma 6, if $\tau<0$ then $Z^{(1)}, Z^{(2)}$, and $Z^{(3)}$ exist and $\left\|Z^{(1)}+Z^{(2)}-2 Z^{(3)}\right\|_{p} \leq C_{0}^{\prime} M^{\prime} b^{(p-1) / p} \sum_{m=0}^{\infty}(1+m)^{2} b^{\frac{m+1}{p} \tau} D_{p}\left(W^{(1)}, W^{(2)}, W^{(3)}\right)^{1 / p}$. 
Proof of Lemma 6. Combining (3.3) with the identity

$$
\prod_{m=l+1}^{n-1} a_{m}=1+\sum_{m=l+1}^{n-1}(-1)^{n-1-m}\left(\prod_{j=l+1}^{m-1} a_{j}\right)\left(a_{m}-1\right)
$$

yields for all integers $b \geq 2$ and $n \geq 1$

$\prod_{i=0}^{n-1} a_{i}^{(1)}+\prod_{i=0}^{n-1} a_{i}^{(2)}-2 \prod_{i=0}^{n-1} a_{i}^{(3)}=\sum_{m=0}^{n-1}\left[f_{m}+\sum_{k=0}^{m-1} f_{k, m}+g_{k, m}+\sum_{k=0}^{m-2} \sum_{l=k+1}^{m-1} g_{k, l, m}\right]$

with

$$
\left\{\begin{array}{l}
f_{m}=\left(\prod_{j=0}^{m-1} a_{j}^{(3)}\right)\left(a_{m}^{(1)}+a_{m}^{(2)}-2 a_{m}^{(3)}\right) \\
f_{k, m}=\left(\prod_{j=0}^{k-1} a_{j}^{(3)}\right)\left(a_{k}^{(1)}+a_{k}^{(2)}-2 a_{k}^{(3)}\right)(-1)^{n-1-m}\left(\prod_{j=k+1}^{m-1} a_{j}^{(1)}\right)\left(a_{m}^{(1)}-1\right) \\
g_{k, m}=\left(\prod_{j=0}^{k-1} a_{j}^{(3)}\right)\left(a_{k}^{(2)}-a_{k}^{(3)}\right)\left(\prod_{j=k+1}^{m-1} a_{j}^{(1)}\right)\left(a_{m}^{(2)}-a_{m}^{(1)}\right)
\end{array}\right.
$$

and

$$
\begin{array}{r}
g_{k, l, m}=\left(\prod_{j=0}^{k-1} a_{j}^{(3)}\right)\left(a_{k}^{(2)}-a_{k}^{(3)}\right)\left(\prod_{j=k+1}^{l-1} a_{j}^{(1)}\right)\left(a_{l}^{(2)}-a_{l}^{(1)}\right)(-1)^{n-1-m} \\
\cdot\left(\prod_{j=l+1}^{m-1} a_{j}^{(2)}\right)\left(a_{m}^{(2)}-1\right) .
\end{array}
$$

Then, the equality

$$
Z_{b^{n}}^{(1)}+Z_{b^{n}}^{(2)}-2 Z_{b^{n}}^{(3)}=\int_{0}^{1}\left(\prod_{i=0}^{n-1} a_{i}^{(1)}(x)+\prod_{i=0}^{n-1} a_{i}^{(2)}(x)-2 \prod_{i=0}^{n-1} a_{i}^{(3)}(x)\right) d x
$$

implies

$$
\begin{aligned}
\| Z_{b^{n}}^{(1)}+Z_{b^{n}}^{(2)}- & 2 Z_{b^{n}}^{(3)} \|_{p} \leq \sum_{m=0}^{n-1}\left(\left\|\int_{0}^{1} f_{m}(x) d x\right\|_{p}+\sum_{k=0}^{m-1}\left\|\int_{0}^{1} f_{k, m}(x) d x\right\|_{p}\right. \\
+ & \left.\left\|\int_{0}^{1} g_{k, m}(x) d x\right\|_{p}+\sum_{k=0}^{m-2} \sum_{l=k+1}^{m-1}\left\|\int_{0}^{1} g_{k, l, m}(x) d x\right\|_{p}\right) .
\end{aligned}
$$

Fix $m \geq 0$ and $h \in\left\{f_{m}, f_{k, m}, g_{k, m}, g_{k, l, m} ; 0 \leq k<l \leq m-1\right\}$. When $m \geq 2$, write for $x \in\left[0, b^{-m}\right]$

$$
h_{i}(x)=\sum_{0 \leq 3 k+i<b^{m}} h\left(x+\frac{3 k+i}{b^{m}}\right) .
$$


One can apply Lemma 2 to the previous sums (for $i=0,1,2$ ), where the role of $V_{i}$ in Lemma 2 is played here by the last term of the product defining $h\left(x+\frac{3 k+i}{b^{m}}\right)$. This, together with Lemma 5 and the fact that $\mathbb{E}\left(\left|a_{k}^{(j)}(x)\right|^{p}\right)=$ $e^{\Lambda\left(C_{b^{k+1}}(x) \backslash C_{b^{k}}(x)\right) \theta_{W^{(j)}}(p)} \leq b^{\tau+p-1}$ for $j \in\{1,2,3\}$ and $k \geq 0$, yield a universal constant $C_{1}>0$, such that for every $x \in\left[0, b^{-m}\right]$ and $0 \leq i \leq 2$

$$
\begin{aligned}
& \mathbb{E}\left(\left|h_{i}(x)\right|^{p}\right) \leq C_{1}\left(M^{\prime}\right)^{p}\left(\prod_{k=0}^{m} e^{\Lambda\left(C_{b^{k+1}}(x) \backslash C_{b^{k}}(x)\right) \max _{j \in\{1,2,3\}} \theta_{W^{(j)}}(p)}\right) \\
& \quad \times D_{p}\left(W^{(1)}, W^{(2)}, W^{(3)}\right)=C_{1}\left(M^{\prime}\right)^{p} b^{(\tau+p-1)(m+1)} D_{p}\left(W^{(1)}, W^{(2)}, W^{(3)}\right) .
\end{aligned}
$$

Then

$$
\begin{aligned}
\mathbb{E}\left|\int_{0}^{1} h(x) d x\right|^{p} & \leq 3^{p-1} b^{-m(p-1)} \int_{0}^{b^{-m}} \sum_{i=0}^{2} \mathbb{E}\left(\left|h_{i}(x)\right|^{p}\right) d x \\
& \leq 3^{p-1} C_{1}\left(M^{\prime}\right)^{p} b^{(p-1)} b^{(m+1) \tau} D_{p}\left(W^{(1)}, W^{(2)}, W^{(3)}\right) .
\end{aligned}
$$

When $m=0$ or $m=1$, a simplest computation yields a similar estimate, and the conclusion is immediate.

The following lemma is already used in Barral ([1]) to study the differentiability of a martingale limit. It is a slightly stronger form of a well known result (see Stein and Zygmund ([25])):

Lemma 7 Let $a<b$ be in $\mathbb{R}$. Let $f$ be a continuous function from $[a, b]$ to $\mathbb{R}$. Assume that there exists a positive function $\psi$ on $\mathbb{R}_{+}$, monotonically decreasing in a neighborhood of 0 , such that $\frac{\psi(h)}{h}$ is integrable near 0 , and for some constant $M>0: \forall j \in \mathbb{N}$ and $0 \leq k<2^{j}-2$

$$
\begin{aligned}
& \mid f\left(a+\frac{k(b-a)}{2^{j}}\right)+ f\left(a+\frac{(k+2)(b-a)}{2^{j}}\right)-2 f\left(a+\frac{(k+1)(b-a)}{2^{j}}\right) \mid \\
& \leq M \frac{b-a}{2^{j}} \psi\left(\frac{b-a}{2^{j}}\right) .
\end{aligned}
$$

Then $f$ is continuously differentiable.

\section{The general case}

This section deals with extensions of the results of Section 3 when $\Lambda$ assumes weaker (or no) invariance properties.

Recall that for $p \geq 1$

$$
\theta_{W}(p)=\mathbb{E}\left(\left|W^{p}\right|\right)-1-p(\mathbb{E}(\Re(W))-1) .
$$


Theorem 6 ( $L^{p}$ convergence) Fix $s>0$.

i) If $\Lambda\left(\cup_{x \in[0, s]} C(x)\right)<\infty$ then almost surely there exists $t_{s} \geq 1$ such that $Z_{t}^{(s)}$ converges, as $t \rightarrow \infty$, to

$$
Z^{(s)}=\int_{0}^{s} e^{-\Lambda(C(x))(\mathbb{E}(W)-1)} \prod_{M \in S \cap C_{t_{s}}(x)} W_{M} d x .
$$

ii) If $p \in] 1,2]$ then for all integers $b \geq 2$ and $n \geq \max \left(0, \frac{\log (s)}{\log (b)}\right)$

$$
\mathbb{E}\left(\left|Z_{s^{-1} b^{n+1}}^{(s)}-Z_{s^{-1} b^{n}}^{(s)}\right|^{p}\right) \leq \frac{12^{p}}{3} I_{n}(s, b, p)
$$

where

$$
I_{n}(s, b, p)=\left(s b^{-n}\right)^{(p-1)} \int_{[0, s]} e^{\Lambda\left(C_{s^{-1} b^{n+1}}(x)\right) \theta_{W}(p)} d x .
$$

Consequently, if $\sum_{n \geq 0} I_{n}^{1 / p}(s, b, p)<\infty$ for some $b \geq 2$, then the martingale $\left(Z_{t}^{(s)}\right)_{t \geq 1}$ converges almost surely and in $L^{p}$ norm to a random variable $Z^{(s)}$ with mean $s$.

iii) If $p=2$ then for every $s>0$ the martingale $\left(Z_{t}^{(s)}\right)_{t \geq 1}$ converges almost surely and in $L^{2}$ norm to a random variable $Z^{(s)}$ if and only if

$$
\int_{[0, s]^{2}} e^{\Lambda(C(u) \cap C(v)) \theta_{W}(2)} d u d v<\infty .
$$

In that case, the $L^{2}$ norm of $Z^{(s)}$ is the above integral.

Proof. $i$ ) It is a consequence of the dominated convergence Theorem and the almost sure finiteness of the set $S \cap \cup_{x \in[0, s]} C(x)$. ii) Adapt the proof of Lemma 3. $i i i)$ It is Lemma 4.ii).

Remark 6. If the convergence in Theorem 6 holds for some integer $b \geq 2$, it holds for every integer $b \geq 2$.

\subsection{Uniform convergence of martingales}

We consider the random functions $\lambda \in U \mapsto W_{k, n}(\lambda)$ introduced in Section 3 and for every $s \geq 0$ we consider the associated family of martingales $\left(Z_{t}^{(s)}(\lambda)\right)_{t \geq 1}, \lambda \in U$.

Theorem 7 The conclusions of Theorem 2 hold after replacing, for every $s>0$, the assumption $\sup _{\lambda \in K} \tau_{W(\lambda), \delta}\left(p_{K}\right)<0$ by

$$
\sum_{n \geq 0}\left(s b^{-n}\right)^{\left(p_{K}-1\right) / p_{K}}\left(\int_{[0, s]} e^{\Lambda\left(C_{s^{-1}} b^{n+1}(x)\right) \sup _{\lambda \in K} \theta_{W(\lambda)}\left(p_{K}\right)} d x\right)^{1 / p_{K}}<\infty
$$

for some (or equivalently for every) integer $b \geq 2$.

The proof is similar to the one of Theorem 2 and uses Theorem 6.ii). 
Now, for $s>0$, let $\left(A_{s}\right)$ be the following assertion:

$\left(A_{s}\right)$ : for some (or equivalently for every) integer $b \geq 2$,

$$
\sup _{(k, x) \in \mathbb{N} \times[0, s]} \Lambda\left(C_{b^{k+1}}(x) \backslash C_{b^{k}}(x)\right)<\infty .
$$

Moreover if $U \subset \mathbb{R}$ then for every bounded open subinterval $I$ of $U$ such that $\bar{I} \subset U$, and every $p_{\bar{I}}>1$, let $\left(A_{s}\left(I, p_{\bar{I}}\right)\right)$ be the assertion:

$\left(A_{s}\left(I, p_{\bar{I}}\right)\right)$ : the mapping $\lambda \mapsto \mathbb{E}\left(|W(\lambda)|^{p_{\bar{I}}}\right)$ is continuous on $\bar{I}$ and

$$
\sum_{m \geq 0} m^{2}\left(b^{-m\left(p_{\bar{I}}-1\right)} \int_{[0, s]} e^{\Lambda\left(C_{s^{-1} b^{m+1}}(x)\right) \sup _{\lambda \in \bar{I}} \theta_{W(\lambda)}\left(p_{\bar{I}}\right)} d x\right)^{1 / p_{\bar{I}}}<\infty
$$

for some (or equivalently for every) integer $b \geq 2$.

Define $\left(H_{1}^{(s)}\right)$ and $\left(H_{2}^{(s)}\right)$ as being respectively the same sequence of assumptions as $\left(H_{1}\right)$ and $\left(H_{2}\right)$ (defined in Section 3.2), except that assumption $i)$ of $\left(H_{1}\right)$ is replaced by $\left(A_{s}\left(I, p_{\bar{I}}\right)\right)$.

Theorem 8 Fix $s>0$ such that $\left(A_{s}\right)$ holds.

1) Assume $\left(H_{1}^{(s)}\right)$. The conclusions of Theorem 3.1) hold.

2) Assume $\left(H_{2}^{(s)}\right)$. The conclusions of Theorem 3.2) hold.

The proof follows path by path the one of Theorem 3 with the following changes.

Fix $b$ and integer $\geq 2$. Let $k_{s}$ be the largest integer less than or equal to $\log s / \log b$ (notice that $k_{s}<0$ if $s<1$ ). It follows from $\left(A_{s}\right)$ that

$$
m_{s}=\sup _{(k, x) \in\left[k_{s}, \infty\right] \times[0, s]} \Lambda\left(C_{s^{-1} b^{k+1}}(x) \backslash C_{s^{-1} b^{k}}(x)\right)<\infty .
$$

Define

$$
\left\{\begin{array}{l}
\varepsilon^{\prime}=\max _{1 \leq i \leq 3} \mathbb{E}\left(\left|W^{(i)}\right|^{p}\right)(\rho-1)+p \max _{1 \leq i, j \leq 3}\left[\mathbb{E}\left(W^{(i)}-W^{(j)}\right)\right] \\
\theta=\max _{1 \leq i \leq 3} \theta_{W^{(i)}}(p) \\
M^{\prime}=\left(\max \left(1, m_{s}^{4}\right) M e^{m_{s} \varepsilon^{\prime}}\right)^{2 / p}
\end{array}\right.
$$

and for all integer $k \geq k_{s}, x \in[0, s]$ and $i \in\{1,2,3\}$ define

$$
\begin{aligned}
& a_{k}^{(i)}(x)=e^{-\Lambda\left(C_{s}-1 b^{k+k_{s}+1}(x) \backslash C_{s}-1 b^{k+k_{s}}(x)\right)\left(\mathbb{E}\left(W^{(i)}\right)-1\right)} \\
& \times \prod_{M \in S \cap C_{s^{-1}} b^{k+k_{s}+1}(x) \backslash C_{s^{-1} b^{k+k_{s}}}(x)} W_{M}^{(i)} .
\end{aligned}
$$


Then the conclusions of Lemma 5 and Corollary 1 can be extended respectively in:

1) for all $k \geq 0$ and all $x \in[0, s]$,

$$
\begin{aligned}
& \mathbb{E}\left(\left|a_{k}^{(1)}(x)+a_{k}^{(2)}(x)-2 a_{k}^{(3)}(x)\right|^{p}\right) \\
& \quad \leq C_{0} \max \left(1, m_{s}^{4}\right) M e^{\Lambda\left(C_{s^{-1}} b^{k+k_{s}+1}(x) \backslash C_{s^{-1} b^{k+k_{s}}}(x)\right)\left(\theta+\varepsilon^{\prime}\right)} D_{p}\left(W^{(1)}, W^{(2)}, W^{(3)}\right)
\end{aligned}
$$

and

2) $\quad\left\|\lim \inf _{n \rightarrow \infty}\left|\left(Z_{s^{-1} b^{n}}^{(s)}\right)^{(1)}+\left(Z_{s^{-1} b^{n}}^{(s)}\right)^{(2)}-2\left(Z_{s^{-1} b^{n}}^{(s)}\right)^{(3)}\right|\right\|_{p}$

$$
\begin{array}{r}
\leq C_{0}^{\prime} M^{\prime} \sum_{m=0}^{\infty}(1+m)^{2}\left(\left(s b^{-m}\right)^{(p-1)} \int_{[0, s]} e^{\Lambda\left(C_{\left.s^{-1} b^{m+1+k_{s}}(x)\right) \theta} d x\right)^{1 / p}}\right. \\
. D_{p}\left(W^{(1)}, W^{(2)}, W^{(3)}\right)^{1 / p} .
\end{array}
$$

\subsection{Properties of the related measures}

In all the section the random variable $W$ is non-negative.

\subsubsection{Local Hölder exponents and dimension}

In this subsection we assume the following property:

$\Lambda$ is invariant by translations in the spatial direction,

i. e.

$$
\Lambda=\ell \otimes \nu
$$

where $\nu$ is a Borel measure on ]0,1]. Moreover, assume that

$$
\sup _{0<\varepsilon \leq 1} \varepsilon \nu([\varepsilon, 1])<\infty .
$$

Then consider $\delta_{1}, \delta_{2}$ and $\widehat{\delta}_{2}$ as defined in Section 1.2. Writing $\int_{1 / s}^{1 / t} y \nu(d y)=$ $\int_{1 / s}^{1 / t} \int_{0}^{y} d x \nu(d y)$ and using Fubini theorem shows that $\sup _{0<\varepsilon \leq 1} \varepsilon \nu([\varepsilon, 1])<$ $\infty$ implies $\widehat{\delta}_{2}<\infty$.

Theorem 9 Assume that $\tau_{W, \widehat{\delta}_{2}}(p)<0$ for some $\left.\left.p \in\right] 1,2\right]$.

i) If $0<\delta_{1} \leq \delta_{2}$ and $\mathbb{P}(W=1)<1$ then $\mathbb{P}\left(\mu_{W} \neq 0\right)>0$ and, with probability one, conditionally on $\mu_{W} \neq 0$, for $\mu_{W}$-almost every $x \in \mathbb{R}_{+}$

$$
\begin{aligned}
0 & <-\tau_{W, \delta_{2}}^{\prime}(1) \leq \lim \inf _{r \rightarrow 0^{+}} \frac{\log \mu_{W}([x-r, x+r])}{\log 2 r} \\
& \leq \lim \sup _{r \rightarrow 0^{+}} \frac{\log \mu_{W}([x-r, x+r])}{\log 2 r} \leq-\tau_{W, \delta_{1}}^{\prime}(1)<1 .
\end{aligned}
$$


Consequently $-\tau_{W, \delta_{2}}^{\prime}(1) \leq \operatorname{dim} \mu_{W} \leq-\tau_{W, \delta_{1}}^{\prime}(1)<1$; in particular $\mu_{W}$ is singular with respect to the Lebesgue measure. Moreover, $\mu_{W}(B)>0 \Rightarrow$ $\operatorname{dim}(B) \geq-\tau_{W, \delta_{2}}^{\prime}(1)$ for $B \in \mathcal{B}\left(\mathbb{R}_{+}\right)$.

If $\delta_{1}=\delta_{2}$ then $\mu_{W}$ and the statistically self-similar measure associated with $\left(W, \Lambda_{\delta_{1}}\right)$ borrow the same dimension.

ii) If $\delta_{2}=0$ then $\mathbb{P}\left(\mu_{W} \neq 0\right)>0$ and, with probability one, conditionally on $\mu_{W} \neq 0$, for $\mu_{W}$-almost every $x \in \mathbb{R}_{+}$

$$
\lim _{r \rightarrow 0^{+}} \frac{\log \mu_{W}([x-r, x+r])}{\log 2 r}=1 .
$$

Consequently, $\operatorname{dim} \mu_{W}=1$. Moreover, $\mu_{W}(B)>0 \Rightarrow \operatorname{dim}(B)=1$ for $B \in \mathcal{B}\left(\mathbb{R}_{+}\right)$.

Theorem 8.2) makes it possible to establish a continuous version of the previous results.

It follows from the invariance of $\Lambda$ under translations in the spatial direction that the assumptions of Theorem 8.2) are fulfilled for some $s>0$ if and only if they are fulfilled for all $s>0$.

Theorem 10 Assume the assumptions of Theorem 8.2) are fulfilled for some $s>0$ and that the $W(\lambda)$ 's are non-negative.

Assume that

$$
\sup _{\lambda \in \bar{I}} \tau_{W(\lambda), \widehat{\delta}_{2}}\left(p_{\bar{I}}\right)<0
$$

for every compact subinterval $\bar{I}$ of $U$ ( $p_{\bar{I}}$ is introduced in $\left(H_{2}^{(s)}\right)$ and do not depend on s here).

Finally, assume that for every compact subset $K$ of $U$ there exists $\gamma_{K}>1$ such that

$$
\sup _{\lambda \in K} \mathbb{E}\left(\left|\frac{d}{d \lambda} W(\lambda)\right| .|\log (W(\lambda))|\right)+\mathbb{E}\left(\sup _{\lambda \in K}(W(\lambda))^{\gamma_{K}}\right)<\infty .
$$

i) If $0<\delta_{2}<\infty$ then, with probability one, the measures $\mu_{W(\lambda)}, \lambda \in U$, are defined simultaneously, and for every $\lambda \in U$, conditionally on $\mu_{W(\lambda)} \neq 0$, for $\mu_{W(\lambda)}$-almost every $x \in \mathbb{R}_{+}$

$$
\lim \inf _{r \rightarrow 0^{+}} \frac{\log \mu_{W(\lambda)}([x-r, x+r])}{\log 2 r} \geq-\tau_{W(\lambda), \delta_{2}}^{\prime}(1)>0 .
$$

Consequently, $\mu_{W(\lambda)}(B)>0 \Rightarrow \operatorname{dim}(B) \geq \tau_{W(\lambda), \delta_{2}}^{\prime}(1)$ for $B \in \mathcal{B}\left(\mathbb{R}_{+}\right)$, and $\operatorname{dim} \mu_{W(\lambda)} \geq \tau_{W(\lambda), \delta_{2}}^{\prime}(1)$.

ii) If $\delta_{2}=0$ then, with probability one, the measures $\mu_{W(\lambda)}, \lambda \in U$, are defined simultaneously, and for every $\lambda \in U$, the conclusions of Theorem 9.ii) hold with $\mu_{W(\lambda)}$ instead of $\mu_{W}$. 
Remark 7. 1) The case $\delta_{2}=\infty$ is excluded because, when $\Lambda$ is invariant by horizontal translations, the sensible way to exploit Theorem 6.ii) is to remark that $\sum I_{n}(s, b, p)$ converge if $\tau_{W, \delta_{2}}(p)<0$, requiring that $\delta_{2}<\infty$. The same remark shows that in Theorem 10, the assumption $\sup _{\lambda \in \bar{I}} \tau_{W(\lambda), \widehat{\delta}_{2}}\left(p_{\bar{I}}\right)<0$ implies that the assumption $i$ ) of $\left(H_{2}^{(s)}\right)$ is fulfilled.

2) Theorem 9 would give estimates on the local Hölder exponents and dimension only for every $\lambda \in U$ almost surely. So deduce Theorem 10 directly from Theorem 9 is not possible.

3) If $\Lambda=\Lambda_{\delta}$, Theorem 9.i) is an extension of Theorem 4 to the case when $W$ vanishes with positive probability.

4) The limsup of the logarithmic density does not appear in the continuous version of Theorem 9.i) because it would involve an uniform control on the moments of negative orders of pieces of the $\mu_{W(\lambda)}$ 's. We are able to obtain such a control only in the self-similar case (see Remark 5).

5) The parameter $\widehat{\delta}_{2}$ will play its main role in the proof of Lemma 8.i). The assumption $\sup _{0<\varepsilon<1} \varepsilon \nu([\varepsilon, 1])<\infty$ is equivalent to saying that $\Lambda\left(B^{I}\right)$ is bounded independently of $I$ if $I$ is a non trivial subinterval of $\mathbb{R}_{+}$with length $\leq 1$.

6) The conclusion of Theorem 9.ii) leads to the problem to decide whether or not $\mu_{W}$ is absolutely continuous with respect to the Lebesgue measure. This is solved under satisfactory conditions in section 4.2.2.

We prove Theorem 10 and give indications for the result concerning the limsup in Theorem 9.i).

Proof of Theorem 10.i). We begin by showing that the measures are defined almost surely simultaneously.

The invariance of $\Lambda$ by translations in the spatial direction allows to reduce the study to the restriction of the $\mu_{W(\lambda)}$ 's to $[0,1]$. These restrictions are still denoted by $\mu_{W(\lambda)}$.

Fix an integer $b \geq 2$. We need to define measures on a $b$-ary tree, and we set up new definitions.

For an integer $m \geq 0$, we denote by $A_{m}$ the set of finite words of length $m$ on the alphabet $\{0, \ldots, b-1\}\left(A_{0}=\{\epsilon\}\right)$.

For $a \in A_{m}$, let $|a|$ denote its length $m$, and let $I_{a}$ denote the closed $b$-adic subinterval of $[0,1]$ naturally encoded by $a$.

Define $A=\bigcup_{m=1}^{\infty} A_{m}$ and $\partial A=\{0, \ldots, b-1\}^{\mathbb{N}} ; A \cup \partial A$ is equipped with the concatenation operation and for $a \in A$, define $C_{a}=a \partial A$, the cylinder generated by $a$. Let $\mathcal{A}$ be the $\sigma$-field generated by the $C_{a}$ 's in $\partial A$. 
Let $\pi$ be the mapping from $\partial A$ to $[0,1]$ defined by $x=x_{1} \ldots x_{i} \ldots \mapsto$ $\sum_{i \geq 1} x_{i} / b^{i}$. Let $\tilde{\ell}$ be the unique measure on $(\partial A, \mathcal{A})$ such that for all $a \in A$, $\tilde{\ell}\left(C_{a}\right)=b^{-|a|}$.

For every $t \geq 1$ and $\lambda \in U$ define $\nu_{W(\lambda), t}$ the measure whose density with respect to $\tilde{\ell}$ is

$$
\frac{d \nu_{W(\lambda), t}}{d \tilde{\ell}}(x)=e^{-\Lambda\left(C_{t}(\pi(x))\right)(\mathbb{E}(W)-1)} \prod_{M \in S \cap C_{t}(\pi(x))} W_{M} .
$$

By construction, with probability one, for all $(t, \lambda) \in[1, \infty[\times U$

$$
\nu_{W(\lambda), t} \circ \pi^{-1}=\mu_{W(\lambda), t} .
$$

As $\partial A$ is totally disconnected, if we show that for every $a \in A, \nu_{W(\lambda), t}\left(C_{a}\right)$ converges almost surely for all $\lambda \in U$ as $t \rightarrow \infty$ to a limit $\nu_{W(\lambda)}\left(C_{a}\right)$, then the additive function $\nu_{W(\lambda)}$ on cylinders will extend in an unique measure on $(\partial A, \mathcal{A})$. Moreover, this measure $\nu_{W(\lambda)}$ will be the weak limit of the $\nu_{W(\lambda), t}$ 's. Then by (4.2) the measures $\mu_{W(\lambda)}$ will be obtained simultaneously from the relation $\nu_{W(\lambda)} \circ \pi^{-1}=\mu_{W(\lambda)}$.

By the invariance property of $\Lambda$ and the fact that $A$ is countable, it suffices to show that $\lambda \mapsto \nu_{W(\lambda), t}\left(C_{a}\right)$ converges for finite words $a$ written with the letter 0 only. In this case if $|a|=k$ and $s=b^{-k}$ then $\lambda \mapsto$ $\nu_{W(\lambda), t}\left(C_{a}\right)=\lambda \mapsto Z_{t}^{(s)}(\lambda)$ which converges uniformly on compact subsets by Theorem 8.2).

Moreover, it follows from the hypotheses that each $\mu_{W(\lambda)}$ is positive with positive probability, since its total mass is the limit of the uniformly integrable martingale $Z_{t}^{(1)}(\lambda)$.

Now we prove the assertion on the liminf of the logarithmic density. We need the following lemma. It involves some definitions introduced in Section 2 and will be proved after Theorems 9 and 11 .

Lemma 8 Assume the assumptions of Theorem 10i). Fix a compact subinterval $\bar{I}$ of $U$. Fix $b \geq 2$ so that $\sup _{\lambda \in \bar{I}} \tau_{W(\lambda), \delta_{2}(b)}\left(p_{\bar{I}}\right)<0$ (see Section 1.2 for the definition of $\left.\delta_{2}(b)\right)$.

i) With probability one, for every $a \in A$, the random variables $\left\|\mu_{W(\lambda)}^{I_{a}}\right\|$, $\lambda \in \bar{I}$, are defined simultaneously and the function $\lambda \mapsto Z^{I_{a}}(\lambda)=b^{|a|}\left\|\mu_{W(\lambda)}^{I_{a}}\right\|$ is of class $C^{1}$ on $\bar{I}$. Moreover

$$
\sup _{a \in A, \lambda \in \bar{I}} \mathbb{E}\left(\left(Z^{I_{a}}(\lambda)\right)^{p_{\bar{I}}}\right)+\sup _{a \in A, \lambda \in \bar{I}} \mathbb{E}\left(\left|\frac{d Z^{I_{a}}}{d \lambda}(\lambda)\right|^{p_{\bar{I}}}\right)<\infty
$$


ii) For $m \geq 1, \varepsilon>0, \eta>0$ and $\lambda \in \bar{I}$ define

$$
\begin{aligned}
f_{m, \varepsilon, \eta}(\lambda)= & e^{-(1+\eta) \Lambda\left(C_{b^{m}}(0)\right)(\mathbb{E}(W(\lambda))-1)} b^{-m \eta\left(\tau_{W(\lambda), \delta_{2}}^{\prime}(1)+\varepsilon\right)} \times \\
& \sum_{a \in A_{m}} Q_{W(\lambda), T^{I_{a}}}^{1+\eta}\left[\sup _{\lambda \in \bar{I}, x \in I_{a}} Q_{W(\lambda), B^{I_{a}}}(x)\right]^{1+\eta} b^{-m(1+\eta)}\left(Z^{I_{a}}(\lambda)\right)^{1+\eta} .
\end{aligned}
$$

If $\eta$ is small enough then $\sum_{m \geq 1} f_{m, \varepsilon, \eta}$ converges almost surely uniformly on $\bar{I}$.

iii) With probability one, for every $\lambda \in \bar{I}$, conditionally on $\nu_{W(\lambda)} \neq 0$, for $\nu_{W(\lambda)}$-almost every $x \in \partial A$,

$$
\lim \inf _{m \rightarrow 0^{+}} \frac{\log \nu_{W(\lambda)}\left(C_{x_{1} \ldots x_{m}}\right)}{\log b^{-m}} \geq-\tau_{W(\lambda), \delta_{2}}^{\prime}(1)>0 .
$$

In particular, the $\nu_{W(\lambda)}$ 's are continuous.

End of the proof. It suffices to establish the result for any compact subinterval contained in $U$, instead of $U$. Fix such an interval, $\bar{I}$. For $m \geq 1, \varepsilon>0$ and $\lambda \in \bar{I}$ define the set

$$
\begin{aligned}
E_{m, \varepsilon}(\lambda)=\left\{x \in \operatorname{supp}\left(\mu_{W(\lambda)}\right)\right. & \cap K_{m} ; \\
& \left.\frac{\log \mu_{W(\lambda)}\left(\left[x-\frac{b^{-m}}{2}, x+\frac{b^{-m}}{2}\right]\right)}{\log \left(b^{-m}\right)} \leq-\tau_{W(\lambda), \delta_{2}}^{\prime}(1)-\varepsilon\right\} .
\end{aligned}
$$

where $K_{m}=\left[b^{-m} / 2,1-b^{-m} / 2\right]$.

$\left(\sup _{\lambda \in \bar{I}}\left\{\tau_{W(\lambda), \widehat{\delta}_{2}}\left(p_{\bar{I}}\right)\right\}<0\right.$ implies $\sup _{\lambda \in \bar{I}}\left\{\tau_{W(\lambda), \delta_{2}}\left(p_{\bar{I}}\right)\right\}<0$, so $\tau_{W(\lambda), \delta_{2}}^{\prime}(1)<$ 0 for every $\lambda \in \bar{I})$.

The conclusion will follow if we prove that for every $\varepsilon>0$, with probability one, for every $\lambda \in \bar{I}$

$$
\sum_{m \geq 1} \mu_{W(\lambda)}\left(E_{m, \varepsilon}(\lambda)\right)<\infty .
$$

Every $E_{m, \varepsilon}(\lambda)$ is empty or can be covered by a finite number of intervals $J_{i}=\left[x_{i}-b^{-m} / 2, x_{i}+b^{-m} / 2\right]$ with $x_{i} \in E_{m, \varepsilon}(\lambda)$. Moreover, we can choose the $J_{i}$ 's so that $\cup J_{i}$ divides into two union of intervals, $\cup J_{k}^{\prime}$ and $\cup J_{\ell}^{\prime \prime}$, with the property that two distinct $J_{k}^{\prime}$ 's or $J_{\ell}^{\prime \prime}$ 's have at most one point in common.

By definition of $E_{m, \varepsilon}(\lambda)$, for every $\eta>0$ and $J_{i}$

$$
\mu_{W(\lambda)}\left(J_{i}\right) \leq \mu_{W(\lambda)}^{1+\eta}\left(J_{i}\right) b^{-m \eta\left(\tau_{W(\lambda), \delta_{2}}^{\prime}(1)+\varepsilon\right)}
$$

Moreover $J_{i}$ is covered by two adjacent $b$-adic subintervals of length $b^{-m}, I_{a}$ and $I_{a^{\prime}}$, so

$$
\mu_{W(\lambda)}^{1+\eta}\left(J_{i}\right) \leq 2^{\eta}\left(\mu_{W(\lambda)}^{1+\eta}\left(I_{a}\right)+\mu_{W(\lambda)}^{1+\eta}\left(I_{a^{\prime}}\right)\right)
$$


By our choice for the covering $\cup J_{i}$, if we choose $b$ as in Lemma 8 we get

$$
\begin{aligned}
\mu_{W(\lambda)}\left(E_{m, \varepsilon}(\lambda)\right) & \leq \sum_{k} \mu_{W(\lambda)}\left(J_{k}^{\prime}\right)+\sum_{\ell} \mu_{W(\lambda)}\left(J_{\ell}^{\prime \prime}\right) \\
& \leq 4.2^{\eta} \sum_{a \in A_{m}} \mu_{W(\lambda)}^{1+\eta}\left(I_{a}\right) b^{-m \eta\left(\tau_{W(\lambda), \delta_{2}}^{\prime}(1)+\varepsilon\right)} \\
& =4.2^{\eta} \sum_{a \in A_{m}} \nu_{W(\lambda)}^{1+\eta}\left(C_{a}\right) b^{-m \eta\left(\tau_{W(\lambda), \delta_{2}}^{\prime}(1)+\varepsilon\right)} \\
& \leq 4.2^{\eta} f_{m, \varepsilon, \eta}(\lambda)
\end{aligned}
$$

where the equality and the last inequality are due respectively to assertion $i$ ii) and proof of Lemma 8. By choosing $\eta$ small enough, we can apply Lemma 8.ii) and conclude.

The assertion on dimension is a direct application of a Billingsley Theorem (Billingsley $([7,136-145]))$.

Proof of Theorem 10.ii). The proof of $i$ ) shows that the conclusion of $i$ ) holds on every compact subset of $U$ for every $\delta_{2}^{\prime}>0$ small enough instead of $\delta_{2}$. Then letting $\delta_{2}^{\prime} \rightarrow 0$ yields the conclusion.

Proof of Theorem 9. The fact that $-\tau_{W, \delta_{1}}^{\prime}(1)<1$ comes from the hypotheses $\mathbb{P}(W=1)<1, \delta_{1}>0$ and the convexity of $\tau_{W, \delta_{1}}$. The result concerning lim inf is obtained as in the proof of Theorem 10. For the lim sup the sets to be considered are the

$E_{m, \varepsilon}^{\prime}=\left\{x \in \operatorname{supp}\left(\mu_{W}\right) \cap K_{m} ; \frac{\log \mu_{W}\left(\left[x-\frac{b^{-m}}{2}, x+\frac{b^{-m}}{2}\right]\right)}{\log \left(b^{-m}\right)} \geq-\tau_{W, \delta_{1}}^{\prime}(1)+\varepsilon\right\}$,

and for every $\eta \in] 0,1[$

$$
\begin{aligned}
\mu_{W}\left(E_{m, \varepsilon}^{\prime}\right) \leq h_{m, \varepsilon, \eta}= & 2 e^{-(1-\eta) \Lambda\left(C_{b^{m}}(0)\right)(\mathbb{E}(W)-1)} b^{-m \eta\left(-\tau_{W, \delta_{1}}^{\prime}(1)+\varepsilon\right)} \\
& \left.\times \sum_{a \in A_{m}} Q_{W, T^{I_{a}}}^{1-\eta} \sup _{x \in I_{a}} Q_{W, B^{I_{a}}}(x)\right)^{1-\eta}\left\|\mu_{W}^{I_{a}}\right\|^{1-\eta} .
\end{aligned}
$$

Then computations similar to those necessary to obtain the control on $\mathbb{E}\left(\left|\frac{d}{d \lambda} f_{m, \varepsilon, \eta}(\lambda)\right|\right)$ in the proof of Lemma 8.ii) show that for every $\varepsilon>0$, if $\eta$ is small enough, $\sum_{m \geq 1} \mathbb{E}\left(h_{m, \varepsilon, \eta}\right)<\infty$, so almost surely $\sum_{m \geq 1} \mu_{W}\left(E_{m, \varepsilon}^{\prime}\right)<\infty$. This yields the assertion on the lim sup.

The assertion on dimension is again a Billingsley Theorem applied to the inequalities on the logarithmic density, and the singularity with respect to the Lebesgue measure comes from the lim sup inequality. 
Proof of Lemma 8.i). Fix $a \in A$. After a change of scale and using the invariance of $\Lambda$, the same approach as in the proof of Theorem 6 shows that for $m \geq 2$ and $p \in] 1,2]$

$$
\mathbb{E}\left(\left|\left\|\mu_{W, b^{m+1}}^{I_{a}}\right\|-\left\|\mu_{W, b^{m}}^{I_{a}}\right\|\right|^{p}\right) \leq \frac{12^{p}}{3} b^{-|a| p} I_{m, a}(p)
$$

where

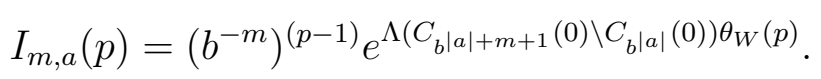

Moreover (4.1) can be extended in

$$
\begin{aligned}
& \left\|\lim \inf _{n \rightarrow \infty}\left|\left\|\mu_{W^{(1)}, b^{n}}^{I_{a}}\right\|+\left\|\mu_{W^{(2)}, b^{n}}^{I_{a}}\right\|-2\left\|\mu_{W^{(3)}, b^{n}}^{I_{a}}\right\|\right|\right\|_{p} \\
& \leq b^{-|a|} C_{0}^{\prime} M^{\prime} \sum_{m=0}^{\infty}(1+m)^{2}\left(\left(b^{-m}\right)^{(p-1)} e^{\Lambda\left(C_{b^{|a|+m+1}}(0) \backslash C_{b|a|}(0)\right) \theta}\right)^{1 / p} \\
& . D_{p}\left(W^{(1)}, W^{(2)}, W^{(3)}\right)^{1 / p} .
\end{aligned}
$$

By definition of $\delta_{2}(b)$, for every $\delta_{2}^{\prime}>\delta_{2}(b)$, if $|a|$ is large enough then with the notations of Lemma 5 and $\delta$ replaced by $\delta_{2}^{\prime}$

$$
\left(b^{-m}\right)^{(p-1)} e^{\Lambda\left(C_{b|a|+m+1}(0) \backslash C_{b|a|}(0)\right) \theta} \leq b^{(m+1) \tau} b^{p-1},
$$

which is independent of $a$. Moreover, if $\delta_{2}^{\prime}$ is chosen close enough to $\delta_{2}(b)$, the hypotheses also imply that $\sup _{\lambda \in \bar{I}} \tau_{W(\lambda), \delta_{2}^{\prime}}\left(p_{\bar{I}}\right)<0$. Then the conclusion comes from arguments similar to those necessary for proving Theorem 8.2).

ii) It is easily seen that it suffices to prove the following: if $\eta$ is small enough then

a) $\sum_{m \geq 1} \mathbb{E}\left(f_{m, \varepsilon, \eta}(\min \bar{I})\right)<\infty$ and

b) $\sum_{m \geq 1} \sup _{\lambda \in \bar{I}} \mathbb{E}\left(\left|\frac{d f_{m, \varepsilon, \eta}}{d \lambda}(\lambda)\right|\right)<\infty$.

Assertion $a$ ) is a consequence of computations leading to $b$ ); so we only prove $b)$.

By the invariance of $\Lambda$ by translation in the spatial direction,

$$
M_{\eta, a}=\mathbb{E}\left[\left(\sup _{\lambda \in \bar{I}, x \in I_{a}} Q_{W(\lambda), B^{I a}}(x)\right)^{1+\eta}\right]
$$

does not depend on $a \in A_{m}$ and we denote this number by $M_{\eta}$. We also define $a_{m}$, the word written with $m$ times the letter 0 and

$$
g_{m, \varepsilon, \eta}(\lambda)=e^{-(1+\eta) \Lambda\left(C_{b} m(0)\right)(\mathbb{E}(W(\lambda))-1)} b^{-m \eta\left(\tau_{W(\lambda), \delta_{2}}^{\prime}(1)+\varepsilon\right)} .
$$


By the invariance property of $\Lambda$ and the independences between random variables

$$
\begin{aligned}
\mathbb{E}\left(\left|\frac{d f_{m, \varepsilon, \eta}}{d \lambda}(\lambda)\right|\right) \leq M_{\eta} b^{-m \eta}\left\{\left|\frac{d g_{m, \varepsilon, \eta}}{d \lambda}(\lambda)\right| \mathbb{E}\left(Q_{W(\lambda), T^{I a_{m}}}^{1+\eta}\right) \mathbb{E}\left[\left(Z^{I_{a_{m}}}(\lambda)\right)^{1+\eta}\right]\right. \\
\quad+g_{m, \varepsilon, \eta}(\lambda) \mathbb{E}\left(\left|\frac{d Q_{W(\lambda), T^{I_{a}}}^{1+\eta}}{d \lambda}(\lambda)\right|\right) \mathbb{E}\left[\left(Z^{I_{a_{m}}}(\lambda)\right)^{1+\eta}\right] \\
\left.+(1+\eta) g_{m, \varepsilon, \eta}(\lambda) \mathbb{E}\left(Q_{W(\lambda), T^{I_{a_{m}}}}^{1+\eta}\right) \mathbb{E}\left(\left|\frac{d Z^{I_{a_{m}}}}{d \lambda}\right|(\lambda)\left(Z^{I_{a_{m}}}(\lambda)\right)^{\eta}\right)\right\} .
\end{aligned}
$$

A computation yields

$$
\begin{aligned}
\left|\frac{d g_{m, \varepsilon, \eta}}{d \lambda}(\lambda)\right| \leq & \left((1+\eta) \Lambda\left(C_{b^{m}}(0)\right)+2 m \eta \delta_{2} \log (b)\right) \\
& \times \mathbb{E}\left(\left|\frac{d W}{d \lambda}(\lambda)\right|(|\log (W(\lambda))|+1)\right) g_{m, \varepsilon, \eta}(\lambda)
\end{aligned}
$$

with $\sup _{\lambda \in \bar{I}} \mathbb{E}\left(\left|\frac{d W}{d \lambda}(\lambda)\right|(|\log (W(\lambda))|+1)<\infty\right.$ by assumption. Moreover if $\eta$ is small enough, then: $i$ ) by the assertion $i$ ) of Lemma 8 all the expectations containing the random variable $Z^{I_{a_{m}}}(\lambda)$ are uniformly bounded over $\left.\bar{I} ; i i\right)$ $M_{\eta}<\infty$ by Lemma 1.ii) applied with $\sup _{\lambda \in \bar{I}}(W(\lambda))^{1+\eta}$ instead of $W$, together with the fact that $\Lambda\left(B^{I}\right)$ is bounded independently of the non-trivial subinterval $I$ of $[0,1]$ (see Remark 7.5)).

Now, using Lemma 1.i) with $G=T^{I_{a_{m}}}$ and $W=W^{1+\eta}(\lambda)$ yields

$$
\mathbb{E}\left(Q_{W(\lambda), T^{I} a_{m}}^{1+\eta}\right)=e^{\Lambda\left(T^{I a_{m}}\right)\left(\mathbb{E}\left(W^{1+\eta}(\lambda)-1\right)\right)} .
$$

Moreover, computing that conditionally on $\# S \cap T^{I_{a_{m}}}=k \geq 1$,

$$
\mathbb{E}\left(\left|\frac{d Q_{W(\lambda), T^{I a_{m}}}^{1+\eta}}{d \lambda}(\lambda)\right|\right) \leq k(1+\eta) \mathbb{E}\left(\left|\frac{d W}{d \lambda}(\lambda)\right| W^{\eta}(\lambda)\right)\left(\mathbb{E}\left(W^{1+\eta}(\lambda)\right)^{k-1}\right.
$$

one obtains

$$
\begin{aligned}
& \mathbb{E}\left(\left|\frac{d Q_{W(\lambda), T^{I a_{m}}}^{1+\eta}}{d \lambda}(\lambda)\right|\right) \\
& \quad \leq(1+\eta) \Lambda\left(T^{I_{a_{m}}}\right) \mathbb{E}\left(\left|\frac{d W}{d \lambda}(\lambda)\right| W^{\eta}(\lambda)\right) e^{\Lambda\left(T^{I a_{m}}\right)\left(\mathbb{E}\left(W^{1+\eta}(\lambda)-1\right)\right)} .
\end{aligned}
$$

It follows from the assumptions of Theorem 9.2) that

$$
\sup _{\lambda \in \bar{I}} \mathbb{E}\left(\left|\frac{d W}{d \lambda}(\lambda)\right| W^{\eta}(\lambda)\right)<\infty
$$

if $\eta$ is small enough. 
By a simple geometrical remark $\Lambda\left(T_{I_{a_{m}}}\right) \leq \Lambda\left(C_{b^{m}}(0)\right)$; so as $0<\delta_{2}<\infty$, for $m$ large enough $\Lambda\left(T^{I_{a_{m}}}\right) \leq \Lambda\left(C_{b^{m}}(0)\right) \leq 2 \delta_{2} m \log (b)$.

Consequently, if $\eta$ is small enough there exists $C_{\eta, \bar{I}}>0$ such that if $m$ is large enough then for all $\lambda \in \bar{I}$ and $\varepsilon>0$

$$
\begin{aligned}
\mathbb{E}\left(\left|\frac{d f_{m, \varepsilon, \eta}}{d \lambda}(\lambda)\right|\right) & \leq C_{\eta, \bar{I}} m \log (b) b^{-m \eta} g_{m, \varepsilon, \eta}(\lambda) e^{\Lambda\left(T^{I a_{m}}\right)\left(\mathbb{E}\left(W^{1+\eta}(\lambda)-1\right)\right)} \\
& \leq C_{\eta, \bar{I}} m \log (b) e^{\Lambda\left(C_{b} m(0)\right) \theta_{W(\lambda)}(1+\eta)} b^{-m \eta\left(1+\tau_{W(\lambda), \delta_{2}}^{\prime}(1)+\varepsilon\right)} .
\end{aligned}
$$

Equation (4.3) can be written

$$
\begin{aligned}
\mathbb{E}\left(\left|\frac{d f_{m, \varepsilon, \eta}}{d \lambda}(\lambda)\right|\right) \leq & C_{\eta, \bar{I}} m \log (b) \\
& . b^{-\eta \varepsilon m} b^{m\left[\tau_{W(\lambda), \delta_{2}}(1+\eta)-\eta \tau_{W(\lambda), \delta_{2}}^{\prime}(1)+\left(\frac{\Lambda\left(C_{b} m(0)\right)}{m \log (b)}-\delta_{2}\right) \theta_{W(\lambda)}(1+\eta)\right]} .
\end{aligned}
$$

Studies of functions show that $\sup _{\lambda \in \bar{I}}\left|\frac{\tau_{W(\lambda), \delta_{2}}(1+\eta)-\eta \tau_{W(\lambda), \delta_{2}}^{\prime}(1)}{\eta}\right| \rightarrow 0$ as $\eta \rightarrow 0^{+}$ and $\theta_{W(\lambda)}(1+\eta) / \eta$ is non-negative and bounded independently of $\lambda \in \bar{I}$ if $\eta$ is small enough.

Since by definition of $\delta_{2}$, for every $\beta>0$, if $m$ is large enough then

$$
\frac{\Lambda\left(C_{b^{m}}(0)\right)}{m \log (b)}-\delta_{2}=\frac{\Lambda\left(C_{b^{m}}(0)\right)}{\Lambda_{1}\left(C_{b^{m}}(0)\right)}-\delta_{2} \leq \beta,
$$

we obtain that for every $\varepsilon>0$ there exists $\eta_{\bar{I}}>0$ and $m_{\bar{I}} \geq 0$ such that if $m \geq m_{\bar{I}}$ then

$$
\sup _{\lambda \in \bar{I}} \mathbb{E}\left(\left|\frac{d f_{m, \varepsilon, \eta_{\bar{I}}}}{d \lambda}(\lambda)\right|\right) \leq C_{\eta_{\bar{I}}, \bar{I}} m \log (b) b^{-\eta_{\bar{I}} \frac{\varepsilon}{2} m}
$$

Similarly

$$
\sup _{\lambda \in \bar{I}} \mathbb{E}\left(f_{m, \varepsilon, \eta_{\bar{I}}}(\lambda)\right) \leq C_{\eta_{\bar{I}}, \bar{I}} b^{-\eta_{\bar{I}} \frac{\varepsilon}{2} m} .
$$

This gives the conclusion.

iii) For $m \geq 1, \varepsilon>0$ and $\lambda \in \bar{I}$ define

$$
F_{m, \varepsilon}(\lambda)=\left\{x \in \operatorname{supp}\left(\nu_{W(\lambda)}\right) ; \frac{\log \nu_{W(\lambda)}\left(C_{x_{1} \ldots x_{n}}\right)}{\log \left(b^{-m}\right)} \leq-\tau_{W(\lambda), \delta_{2}}^{\prime}(1)-\varepsilon\right\} .
$$

The set $F_{m, \varepsilon}(\lambda)$ is empty or is covered by cylinders of the $m^{\text {th }}$ generation, the $C^{(i)}$ 's, satisfying by construction for every $\eta>0$ the inequality

$$
\nu_{W(\lambda)}\left(C^{(i)}\right) \leq \nu_{W(\lambda)}^{1+\eta}\left(C^{(i)}\right) b^{-m \eta\left(\tau_{W(\lambda), \delta_{2}}^{\prime}(1)+\varepsilon\right)} .
$$


So

$$
\nu_{W(\lambda)}\left(F_{m, \varepsilon}(\lambda)\right) \leq \sum_{i} \nu_{W(\lambda)}\left(C^{(i)}\right) \leq \sum_{a \in A_{m}} \nu_{W(\lambda)}^{1+\eta}\left(C_{a}\right) b^{-m \eta\left(\tau_{W(\lambda), \delta_{2}}^{\prime}(1)+\varepsilon\right)} .
$$

Now, by using Proposition 3 and the fact that by construction for all $a \in A_{m}$, $\nu_{W(\lambda)}\left(C_{a}\right)=\lim _{t \rightarrow \infty} \mu_{W(\lambda), t}\left(I_{a}\right)$ one obtains

$$
\sum_{a \in A_{m}} \nu_{W(\lambda)}^{1+\eta}\left(C_{a}\right) b^{-m \eta\left(\tau_{W(\lambda), \delta_{2}}^{\prime}(1)+\varepsilon\right)} \leq f_{m, \varepsilon, \eta}(\lambda)
$$

By $i i)$ this implies that for every $\varepsilon>0$, with probability one,

$$
\sum_{m \geq 1} \nu_{W(\lambda)}\left(F_{m, \varepsilon}(\lambda)\right)<\infty \text { for all } \lambda \in \bar{I}
$$

This yields the conclusion.

\subsubsection{Singularity and absolute continuity}

The assertion $\mathrm{ii}$ ) of Theorem 9 leads naturally to the research of conditions for $\mu_{W}$ to be absolutely continuous with respect to the Lebesgue measure. Proposition 4 gives such conditions in the general case.

Then (Theorem 11) in the context of Section 4.2.1, we give a necessary and sufficient condition on $\Lambda$ for two such measures constructed simultaneously to be mutually singular, and this yields (Corollary 2) a necessary and sufficient condition on $\Lambda$ for $\mu_{W}$ to be absolutely continuous with respect to the Lebesgue measure.

Proposition 4 Fix $s>0$.

i) If $\Lambda\left(\cup_{x \in[0, s]} C(x)\right)<\infty$ then $\mu_{W}$ is absolutely continuous with respect to the Lebesgue measure.

ii) Assume

$$
\int_{[0, s]^{2}} e^{\Lambda(C(u) \cap C(v)) \theta_{W}(2)} d u d v<\infty .
$$

For all $k \geq 0$ the integral $\int_{[0, s]^{2}} e^{\Lambda(C(u) \cap C(v)) \theta_{W}(2)} e^{\frac{2 i k \pi}{s}(u-v)} d u d v$ is non-negative and if

$$
\sum_{k \geq 0} \int_{[0, s]^{2}} e^{\Lambda(C(u) \cap C(v)) \theta_{W}(2)} e^{\frac{2 i k \pi}{s}(u-v)} d u d v<\infty
$$

then with probability one the restriction of $\mu_{W}$ to $[0, s]$ is absolutely continuous with respect to the Lebesgue measure, and its density is in $L^{2}([0, s])$. 
Proof. $i)$ Use Theorem 6.i).

ii) For $k \in \mathbb{Z}$ and $\mu \in\left\{\mu_{W}, \mu_{W, t} ; t \geq 1\right\}$ define $c_{k, s}(\mu)=\int_{[0, s]} e^{\frac{2 i \pi}{s} x} d \mu(x)$. By construction of $\mu_{W}$ and Lemma 4

$$
c_{k, s}\left(\mu_{W}\right)=\lim _{t \rightarrow \infty} c_{k, s}\left(\mu_{W, t}\right)
$$

and

$$
\mathbb{E}\left(\left|c_{k, s}\left(\mu_{W}\right)\right|^{2}\right)=\int_{[0, s]^{2}} e^{\Lambda(C(u) \cap C(v)) \theta_{W}(2)} e^{\frac{2 i k \pi}{s}(u-v)} d u d v .
$$

It follows that if the sum in the statement converges, then with probability one $\sum_{k \in \mathbb{Z}}\left|c_{k, s}\left(\mu_{W}\right)\right|^{2}$ is finite and we have the conclusion.

Now let $\left(W, W^{\prime}\right)$ be a random integrable vector in $\mathbb{R}_{+}^{2}$. With a sequence $\left(W_{k, n}, W_{k, n}^{\prime}\right)_{k, n \geq 1}$ of independent copies of $\left(W, W^{\prime}\right)$ chosen independently of the Poisson point process $S$, one constructs simultaneously the measures $\mu_{W}$ and $\mu_{W^{\prime}}$.

Theorem 11 i) Assume that $\Lambda$ is invariant by translation in the spatial direction and that $\Lambda(C(0))=\infty$, that is if $\Lambda=\ell \otimes \nu, \int_{0}^{1} y \nu(d y)=\infty$.

If $\mathbb{P}\left(W=W^{\prime}\right)<1$ and $\mathbb{P}\left(\left\{\mu_{W} \neq 0 \neq \mu_{W^{\prime}}\right\}\right)>0$ then almost surely, conditionally on $\left\{\mu_{W} \neq 0 \neq \mu_{W^{\prime}}\right\}$, the measures $\mu_{W}$ and $\mu_{W^{\prime}}$ are mutually singular.

ii) Fix $s>0$. If $\Lambda\left(\cup_{x \in[0, s]} C(x)\right)<\infty$ then with probability one the measures $\mu_{W}$ and $\mu_{W}$ are absolutely continuous with respect to the Lebesgue measure on $[0, s]$.

Corollary 2 Assume hypotheses of Theorem 9.ii) and $\mathbb{P}(W=1)<1$.

If $\Lambda(C(0))=\infty$ then almost surely, conditionally on $\left\{\mu_{W} \neq 0\right\}, \operatorname{dim} \mu_{W}=1$ but $\mu_{W}$ and the Lebesgue measure are mutually singular.

If $\Lambda(C(0))<\infty$ then $\mu_{W}$ is almost surely absolutely continuous with respect to the Lebesgue measure.

Remark 8. $\mathbb{P}\left(\left\{\mu_{W} \neq 0 \neq \mu_{W^{\prime}}\right\}\right)>0$ holds for example when $\mathbb{P}\left(\left\{\mu_{V} \neq 0\right\}\right)>0$ for $V \in\left\{W, W^{\prime}\right\}$ and $W$ or $W^{\prime}$ is almost surely positive. Indeed, under the condition $\mathbb{P}(W>0)=1$, it is easily seen that $\left\{\mu_{W} \neq 0\right\}$ is a tail event, so $\mathbb{P}\left(\left\{\mu_{W} \neq 0\right\}\right)=1$ if $\mathbb{P}\left(\left\{\mu_{W} \neq 0\right\}\right)>0$.

Proof of Theorem 11. i) By the invariance property of $\Lambda$ we can make the study on the restriction of $\mu_{W}$ and $\mu_{W^{\prime}}$ to $[0,1]$. We estimate the Hellinger distance between the probability measures $\mu_{W} / Z^{(1)}$ and $\mu_{W^{\prime}} / Z^{\prime(1)}$ conditionally on $\left\{Z^{(1)}=\left\|\mu_{W}\right\| \neq 0 \neq\left\|\mu_{W^{\prime}}\right\|=Z^{\prime(1)}\right\}$ (this tool is also used in 
Liu and Rouault ([19]) to prove the mutual singularity of two multiplicative measures on the boundary of a branching tree). By Theorem 2.5.21 of Dacunha-Castelle and Duflo $([9])$, these measures are mutually singular conditionally on $\left\{Z^{(1)} \neq 0 \neq Z^{(1)}\right\}$ if and only if for some integer $b \geq 2$, conditionally on $\left\{Z^{(1)} \neq 0 \neq Z^{\prime(1)}\right\}$

$$
\lim _{n \rightarrow \infty} d_{n}=\frac{1}{\sqrt{Z^{(1)} Z^{\prime(1)}}} \sum_{k=0}^{b^{n-1}} \sqrt{\mu_{W}\left(\left[\frac{k}{b^{n}}, \frac{k+1}{b^{n}}[) \mu_{W^{\prime}}\left(\left[\frac{k}{b^{n}}, \frac{k+1}{b^{n}}\lceil)\right.\right.\right.\right.}=0
$$

(the Hellinger distance between $\mu_{W} / Z^{(1)}$ and $\mu_{W^{\prime}} / Z^{\prime(1)}$ is then $1-\lim _{n \rightarrow \infty} d_{n}=1$ ).

We only have to prove that $\lim _{n \rightarrow \infty} \mathbb{E}\left(d_{n} \sqrt{Z^{(1)} Z^{\prime(1)}}\right)=0$, since the sequence $d_{n}, n \geq 1$, is non increasing .

Denote by $I$ the interval $\left[0, \frac{1}{b^{n}}[\right.$. By the invariance property of $\Lambda$,

$$
\mathbb{E}\left(d_{n} \sqrt{Z^{(1)} Z^{\prime(1)}}\right)=b^{n} \mathbb{E}\left(\sqrt{\mu_{W}(I) \mu_{W^{\prime}}(I)}\right)
$$

and by using Proposition 3 and Lemma 1

$$
\begin{aligned}
& \frac{\mathbb{E}\left(\sqrt{\mu_{W}(I) \mu_{W^{\prime}}(I)}\right)}{b^{-n} e^{-\Lambda\left(C_{b^{n}}(0)\right) \mathbb{E}\left(\frac{W+W^{\prime}}{2}-1\right)} e^{\Lambda\left(T^{I}\right)\left(\mathbb{E}\left(\sqrt{W W^{\prime}}\right)-1\right)} \mathbb{E}\left(\sqrt{Z_{1} Z_{1}^{\prime}}\right)} \\
& \leq \mathbb{E}\left(\sqrt{\sup _{I} Q_{W, B^{I}}(x) \sup _{I} Q_{W^{\prime}, B^{I}}(x)}\right)
\end{aligned}
$$

By Lemma 1.ii)c), since $\delta_{2}<\infty$, the right hand side of the inequality is bounded independently of $n$ by a constant $K>0$. We saw in the proof of Lemma 8 that $\Lambda\left(T^{I}\right) \leq \Lambda\left(C_{b^{n}}(0)\right)$. So

$$
\mathbb{E}\left(d_{n} \sqrt{Z^{(1)} Z^{\prime(1)}}\right) \leq K \mathbb{E}\left(\sqrt{Z^{(1)} Z^{\prime(1)}}\right) e^{\Lambda\left(C_{b^{n}}(0)\right)\left[\mathbb{E}\left(\sqrt{W W^{\prime}}\right)-\mathbb{E}\left(\frac{W+W^{\prime}}{2}\right)\right]}
$$

and the right hand side tends to 0 . Indeed, $\Lambda\left(C_{b^{n}}(0)\right)$ tends to infinity $(\Lambda(C(0))=\infty)$, the hypothesis $\mathbb{P}\left(W=W^{\prime}\right)<1$ implies

$\mathbb{E}\left(\sqrt{W W^{\prime}}\right)-\mathbb{E}\left(\frac{W+W^{\prime}}{2}\right)<0$, and $\mathbb{E}\left(\sqrt{Z^{(1)} Z^{\prime(1)}}\right)^{2} \leq \mathbb{E}\left(Z^{(1)}\right) \mathbb{E}\left(Z^{\prime(1)}\right) \leq 1$.

ii) Left to the reader.

Remark 9. It is also possible to derive Theorem 11 from the theory developed in Fan ([11]). 


\section{Limit Theorems for $s \mapsto Z^{(s)}$ when $W \geq 0$}

In this section we point out the following interesting fact: when $\Lambda$ is invariant by translation in the spatial direction and for every $s \geq 0$ the r. v.'s $Z^{(s)}$ can be interpreted a.s. as $\mu_{W}([0, s])$ for some r. v. $W \geq 0$, the $Z^{(s)}$ 's are in fact defined a.s. simultaneously. Then we give natural limit theorems for the process with stationary increments $s \geq 0 \mapsto Z^{(s)}$.

With the notations of Section 4.2 we assume that $\Lambda$ is invariant by translation in the spatial direction, $\delta_{2}<\infty$ and $W \geq 0$.

If $\left\{W, W^{\prime}\right\}$ is a pair of non negative r. v.'s in $L^{2}$, we define

$$
\sigma_{W, W^{\prime}}^{2}=\mathbb{E}\left(\int_{[0,1]^{2}} e^{\Lambda(C(u) \cap C(v))\left[\mathbb{E}\left(W W^{\prime}\right)-\mathbb{E}\left(W+W^{\prime}\right)+1\right]} d u d v\right)-1 .
$$

It will be seen that $\sigma_{W, W}^{2}$ is always non negative and we write $\sigma_{W}$ for its square root.

The following results are of the strong law of large numbers and the law of the iterated logarithm type for $\left(Z^{(s)}\right)_{s \geq 0}$.

Theorem 12 Assume the hypotheses of Theorem 9.i) or 9.ii).

i) With probability one, the $Z^{(s)}$ 's, $s \geq 0$, are defined simultaneously and the function $s \mapsto Z^{(s)}$ is continuous and non decreasing.

Moreover the increments of $\left(Z^{(s)}\right)_{s \geq 0}$ are stationary and

$$
\lim _{s \rightarrow \infty} \frac{Z^{(s)}}{s}=1
$$

ii) Assume that $\tau_{W, \delta_{2}}(2)<0$. Then $\sigma_{W}^{2}<\infty$ and with probability one,

$$
\limsup _{s \rightarrow \infty} \frac{\left|Z^{(s)}-s\right|}{(2 s \log \log (s))^{1 / 2}} \leq \sqrt{3} \sigma_{W} .
$$

Proof. i) It follows from the result on the logarithmic density of $\mu_{W}$ in Theorem 9 that $\mu_{W}$ is almost surely continuous. This implies that almost surely, for every $s>0, \lim _{t \rightarrow \infty} \mu_{W, t}([0, s])=\mu_{W}([0, s])$. By definition of $Z^{(s)}$, this yields almost surely the existence of all the $Z^{(s)}$ 's simultaneously, and then $s \mapsto Z_{s}=\mu_{W}([0, s])$ is continuous and non decreasing.

The property of the increments of $\left(Z^{(s)}\right)_{s \geq 0}$ to be stationary is a direct consequence of the invariance of $\Lambda$ by horizontal translations.

The behavior of $Z^{(s)} / s$ will be a consequence of its continuous version in Theorem 13.i).

ii) It is a consequence of the proof of Theorem 13.ii). 
Theorem 12 possesses the following uniform version:

Theorem 13 Assume the hypotheses of Theorem 10.i) or 10.ii).

i) With probability one, the functions $\lambda \in U \mapsto Z^{(s)}(\lambda), s \geq 0$, are defined simultaneously and $Z^{(s)}(\lambda) / s$ converges uniformly to 1 as $s \rightarrow \infty$ on the compact subsets of $U$.

ii) Fix a compact subinterval $K$ of $U$ and assume that $p_{K}=2$. Define $\sigma(K)=\sup _{\lambda \in K} \sigma_{W(\lambda)}$ and $\mathcal{Z}_{s}: \lambda \in K \mapsto Z^{(s)}(\lambda)-s$ for $s \geq 0$.

By construction $\mathcal{K}=\left\{f \in C(K) ; f=\mathbb{E}\left(\xi \mathcal{Z}_{1}\right), \xi \in L^{2}(\Omega), \mathbb{E}\left(\xi^{2}\right) \leq 1\right\}$ is defined, and with probability one

$$
\begin{gathered}
\lim _{\sup _{s \rightarrow \infty}} \frac{\left\|\mathcal{Z}_{s}\right\|_{\infty}}{(2 s \log \log (s))^{1 / 2}} \leq \sqrt{3} \sigma(K), \\
\lim _{s \rightarrow \infty} \inf _{f \in \mathcal{K}}\left\|\frac{\mathcal{Z}_{s}}{(2 s \log \log (s))^{1 / 2}}-\sqrt{3} f\right\|_{\infty}=0 .
\end{gathered}
$$

Consequently, the set of the cluster points of the family $\left(\mathcal{Z}_{s} /(2 s \log \log (s))^{\frac{1}{2}}\right)_{s \geq 0}$ in the space $\left(C(K),\|\|_{\infty}\right)$, as $s$ tends to $\infty$, is contained in the set $\sqrt{3} \mathcal{K}=$ $\{\sqrt{3} f ; f \in \mathcal{K}\}\left(\|f\|_{\infty}\right.$ denotes $\sup _{\lambda \in K} \mid f(\lambda \mid)$.

Proof. $i$ ) The fact that the functions are defined simultaneously is a consequence of Theorem 10 and the arguments in the proof of Theorem 12.

We give an elementary proof of the uniform behavior of $Z^{(s)} / \mathrm{s}$, but the reader can also use the version of the strong law of large numbers for Banach space valued random variables in Ledoux and Talagrand ([18, Corollary 7.10]).

As each function $s \mapsto Z^{(s)}(\lambda)$ is non decreasing, it is enough to show that for every compact subinterval $K$ of $U, \lim _{m \rightarrow \infty} \sup _{\lambda \in K}\left|\frac{Z^{(3 m)}(\lambda)}{3 m}-1\right|=0$ with probability one ( $m$ belongs to $\mathbb{N}$ ).

Fix such a $K$. Then for $m \geq 0, \lambda \in K$ and $i \in\{0,1,2\}$ define

$$
Y_{m}^{i}(\lambda)=Z^{(3 m+i+1)}(\lambda)-Z^{(3 m+i)}(\lambda) .
$$

For each $i \in\{0,1,2\}$, the sets $T_{[3 m+i, 3 m+i+1]}, m \geq 0$, are mutually non overlapping. By using the independence between r. v.'s and the proof of Theorem 8, we see that the sequence $\left(\lambda \in K \mapsto S_{m}^{i}(\lambda)=\frac{1}{m} \sum_{k=0}^{m-1} Y_{k}^{i}(\lambda)\right)_{m \geq 1}$ is a bounded martingale in $\mathcal{W}^{1, p_{K}}(\operatorname{int}(K))$, which converges in $\mathcal{W}^{1, p_{K}}(\operatorname{int}(K))$, almost surely and in $L^{p_{K}}$ norm.

Moreover the $\lambda \in K \mapsto S_{m}^{i}(\lambda)$ 's are continuous (by hypothesis), so they converge almost surely uniformly on $K$.

Now, for each $\lambda \in K$, the increments $Z^{(3 m+i+1)}(\lambda)-Z^{(3 m+i)}(\lambda), m \geq 0$, are non-negative independent and identically distributed r. v.'s with mean 1 , so the strong law of large numbers yields almost surely $\lim _{m \rightarrow \infty} S_{m}^{i}(\lambda)=1$. 
It follows from this that almost surely the function $\lim _{m \rightarrow \infty}(\lambda \in K \mapsto$ $\left.S_{m}^{i}(\lambda)\right)$ is equal to 1 on a dense countable subset of $K$, and so it is identically equal to 1 since it is continuous.

Thus, almost surely, $\lim _{m \rightarrow \infty} \sup _{\lambda \in K}\left|S_{m}^{i}(\lambda)-1\right|=0$. As for $m \geq 1$, $\frac{Z^{(3 m)}(\lambda)}{3 m}-1=\frac{1}{3} \sum_{i=0}^{2}\left(S_{m}^{i}(\lambda)-1\right)$, we get the conclusion.

ii) We shall apply an abstract result (Theorem 8.11 in Ledoux and Talagrand [18]) on the law of the iterated logarithm for Banach spaces valued random variables.

By Theorems 6.iii) and 8, $p_{K}=2$ implies that $\mathbb{E}\left(\left[Z^{(1)}(\lambda)\right]^{2}\right)<\infty$ for every $\lambda \in K$. Then a computation similar to the one in the proof of Lemma 4 yields

$$
\mathbb{E}\left[\left(Z^{(1)}(\lambda)-1\right)\left(Z^{(1)}\left(\lambda^{\prime}\right)-1\right)\right]=\mathbb{E}\left[\mathcal{Z}_{(1)}(\lambda) \mathcal{Z}_{1}\left(\lambda^{\prime}\right)\right]=\sigma_{W(\lambda), W\left(\lambda^{\prime}\right)}^{2}
$$

for every $\left(\lambda, \lambda^{\prime}\right) \in K^{2}$. This implies that

$$
\begin{array}{rl}
\sup _{\nu \in C(K)^{\prime},\|\nu\| \leq 1} & \mathbb{E}\left[\left(\int_{K} \mathcal{Z}_{1}(\lambda) \nu(d \lambda)\right)^{2}\right] \\
& =\sup _{\nu \in C(K)^{\prime},\|\nu\| \leq 1} \int_{K^{2}} \mathbb{E}\left[\mathcal{Z}_{1}(\lambda) \mathcal{Z}_{1}\left(\lambda^{\prime}\right)\right] \nu(d \lambda) \nu\left(d \lambda^{\prime}\right)=\sigma^{2}(K) .
\end{array}
$$

Moreover, by Theorem $8, \mathcal{Z}_{1}$ is of finite $H^{1}=\mathcal{W}^{1,2}(\operatorname{int}(K))$ norm, so

$$
\mathbb{E}\left[\left\|\mathcal{Z}_{1}\right\|_{H^{1}}^{2} / \log \log \left(\max \left(3,\left\|\mathcal{Z}_{1}\right\|_{H^{1}}\right)\right)\right]<\infty .
$$

As the space $H^{1}$ is of type 2 , by Lemma 8.7 of Ledoux and Talagrand ([18]) (applied with $X=\mathcal{Z}_{1}$ in the separable Banach space $H^{1}$ ), with the notations of the proof of $i$ ) this implies that for $i \in\{0,1,2\}, \frac{\sum_{k=0}^{m-1} Y_{k}^{i}(\lambda)-m}{(2 m \log \log (m))^{1 / 2}}$ tends to 0 in probability in $H^{1}$ as $m$ tends to $\infty$.

Moreover, as \|\|$_{\infty}$ is controlled by \|\|$_{H^{1}}$ for the functions considered here, the previous convergence in probability holds in $\left(C(K),\|\|_{\infty}\right)$, (5.1) holds also with \|\|$_{\infty}$ instead of \|\|$_{H^{1}}$, and $\mathbb{E}\left(\left\|\mathcal{Z}_{1}\right\|_{\infty}^{2}\right)<\infty$, allowing to define the set $\mathcal{K}$.

Now for every $i \in\{0,1,2\}$ define the following sequence of independent copies of $\mathcal{Z}_{1}$

$$
\left(\mathcal{Y}_{m}^{i}: \lambda \in K \mapsto Y_{m}^{i}(\lambda)-1\right)_{m \geq 0}
$$

By Theorem 8.11 of Ledoux and Talagrand ([18]), almost surely, for every $i \in\{0,1,2\}$

$$
\begin{array}{r}
\limsup _{m \rightarrow \infty} \frac{\left\|\sum_{k=0}^{m-1} \mathcal{Y}_{k}^{i}\right\|_{\infty}}{(2 m \log \log (m))^{1 / 2}}=\sigma(K), \\
\lim _{m \rightarrow \infty} \inf _{f \in \mathcal{K}}\left\|\frac{\sum_{k=0}^{m-1} \mathcal{Y}_{k}^{i}}{(2 m \log \log (m))^{1 / 2}}-f\right\|_{\infty}=0,
\end{array}
$$


and the set of the cluster points of the family $\left(\frac{\sum_{k=0}^{m-1} \mathcal{Y}_{k}^{i}}{(2 m \log \log (m))^{1 / 2}}\right)_{m \geq 0}$ in $C(K)$, as $m$ tends to $\infty$, is equal to $\mathcal{K}$.

The conclusion comes from the equality $\mathcal{Z}_{3 m}=\sum_{i \in\{0,1,2\}} \sum_{k=0}^{m-1} \mathcal{Y}_{k}^{i}$ and the fact that the mappings $s \geq 0 \mapsto Z^{(s)}(\lambda)$ are non decreasing.

Remark 10. The covariance structure of the r.v. $\mathcal{Z}_{1}$ is the following: if $\nu$ and $\nu^{\prime}$ are two measures in $C(K)^{\prime}$, then

$$
\mathbb{E}\left(\left[\int_{K} \mathcal{Z}_{1}(\lambda) \nu(d \lambda)\right]\left[\int_{K} \mathcal{Z}_{1}(\lambda) \nu^{\prime}(d \lambda)\right]\right)=\int_{K^{2}} \sigma_{W(\lambda), W\left(\lambda^{\prime}\right)}^{2} \nu(d \lambda) \nu^{\prime}\left(d \lambda^{\prime}\right) .
$$

\section{References}

[1] BARral, J.: Differentiability of multiplicative processes related to branching random walks. Ann. Inst. H. Poincaré Probab. Statist. 36 (2000), no. 4, 407-417.

[2] Barral, J.: Continuity of the multifractal spectrum of a random statistically self-similar measure. J. Theoret. Probab. 13 (2000), no. 4, 1027-1060.

[3] Barral, J.: Generalized vector multiplicative cascades. Adv. in Appl. Probab. 33 (2001), 874-895.

[4] Barral, J. And Mandelbrot, B.B.: Multifractal product of cylindrical pulses. Probab. Theory Related Fields 124 (2002), no. 3, 409-430 (and also a different version in Cowles Foundation Discussion paper no. 1287, http://cowles.econ.yale.edu/).

[5] Biggins, J.D.: Uniform convergence of martingales in the one-dimensional branching random walk. In Selected Proceedings of the Sheffield Symposium on Applied Probability (Sheffield, 1989), 159-173. IMS Lecture Notes Monogr. Ser. 18. Inst. Math. Statist., Hayward, CA, 1991.

[6] Biggins, J.D.: Uniform convergence of martingales in the branching random walk. Ann. Probab. 20 (1992), 137-151.

[7] Billingsley, P.: Ergodic Theory and Information, Wiley, N. York, 1965.

[8] Brezis, H.: Analyse fonctionnelle, Théorie et applications. Masson, Paris, 1983.

[9] Dacunha-Castelle, D. And Duflo, M.: Probabilités et statistiques. Tome 2. Problèmes à temps mobile. Collection Mathématiques Appliquées pour la Maîtrise. Masson, Paris, 1983.

[10] Diestel, J. and Uhl, J.J.: Vector measures. Mathematical Surveys 15. American Mathematical Society, Providence, R.I., 1977.

[11] FAN, A.: Equivalence et orthogonalité des mesures aléatoires engendrées par martingales positives homogènes. Studia Math. 98 (1991), 249-266.

[12] FAN, A.: How many intervals cover a point in Dvoretzky covering?. Israel J. Math 131 (2002), 157-184. 
[13] Joffe, A., Le Cam, L. And Neveu, J.: Sur la loi des grands nombres pour les variables aléatoires de Bernouilli attachées à un arbre dyadique. C. R. Acad. Sci. Paris 278 (1973), 963-964.

[14] Kahane, J.-P.: Sur le chaos multiplicatif. Ann. Sci. Math. Québec 9 (1985), 105-150.

[15] Kahane, J.-P.: Positive martingales and random measures. Chinese Ann. of Math. Ser B. 8 (1987), no. 1, 1-12.

[16] Kahane, J.-P.: Produits de poids aléatoires et indépendants et applications. In Fractal geometry and analysis (Montreal, PQ, 1989), 277-324. NATO Adv. Sci. Inst. Ser. C Math. Phys. Sci. 346. Kluwer Acad. Publ., Dordrecht, 1991.

[17] Kahane, J.-P. And Peyrière, J.: Sur certaines martingales de Benoît Mandelbrot. Advances in Math. 22 (1976), no. 2, 131-145.

[18] Ledoux, M. And Talagrand, M.: Probability in Banach spaces. Springer-Verlag, 1991.

[19] Liu, Q. And Rouault, A.: On two measures defined on the limit set of a Galton-Watson tree. In Classical and modern branching processes (Minneapolis, MN, 1994), 187-201. IMA Vol. Math. Appl. 84. Springer, New York, 1997.

[20] Mandelbrot B. B.: Renewal sets and random cutouts. Z. Wahrscheinlichkeitstheorie und Verw. Gebiete 22 (1972), 145-157.

[21] Mandelbrot, B. B.: Multiplications aléatoires itérées et distributions invariantes par moyennes pondérées. C. R. Acad. Sci. Paris 278 (1974), $355-358$

[22] Mandelbrot B. B.: Multifractal products of pulses. Unpublished memorandum, 1996.

[23] Mandelbrot, B. B., Fisher, A. And Calvet, L.: A multifractal model of asset returns. Cowles Foundation Discussion Paper No 1164, 1997.

[24] Neveu J.: Martingales à temps discret. Masson, Paris, 1972.

[25] Stein, E.M. And Zygmund, A.: Smoothness and differentiability of functions. Ann. Univ. Sci. Budapest, Eötvös Sect. Math., 3-4, 1960/1961, 295-307.

[26] von Bahr, B. And Esseen, C.-G.: Inequalities for the $r$ th absolute moment of a sum of random variables $1 \leq r \leq 2$. Ann. Math. Statist. 36 (1965), 299-303.

Recibido: 20 de junio de 2001

Julien Barral

Projet Fractales

INRIA Rocquencourt, domaine de Voluceau-Rocquencourt, B.P. 105

78153 Le Chesnay Cedex, France. julien.barral@inria.fr 